\title{
Observations of the response time of high-latitude ionospheric convection to variations in the interplanetary magnetic field using EISCAT and IMP-8 data
}

\author{
H. Khan, S. W. H. Cowley \\ Department of Physics and Astronomy, University of Leicester, University Road, Leicester LE1 7RH, UK
}

Received: 11 December 1998 / Revised: 2 June 1999 / Accepted: 9 June 1999

\begin{abstract}
We have combined $\sim 300 \mathrm{~h}$ of tristatic measurements of the field-perpendicular $\mathrm{F}$ region ionospheric flow measured overhead at Tromsø by the EISCAT UHF radar, with simultaneous IMP-8 measurements of the solar wind and interplanetary magnetic field (IMF) upstream of the Earth's magnetosphere, in order to examine the response time of the ionospheric flow to changes in the north-south component of the IMF $\left(B_{z}\right)$. In calculating the flow response delay, the time taken by field changes observed by the spacecraft to first effect the ionosphere has been carefully estimated and subtracted from the response time. Two analysis methods have been employed. In the first, the flow data were divided into $2 \mathrm{~h}$-intervals of magnetic local time (MLT) and cross-correlated with the "half-wave rectifier" function $V^{2} B_{s}$, where $V$ is the solar wind speed, and $B_{s}$ is equal to IMF $B_{z}$ if the latter is negative, and is zero otherwise. Response delays, determined from the time lag of the peak value of the cross-correlation coefficient, were computed versus MLT for both the east-west and north-south components of flow. The combined data set suggests minimum delays at $\sim 1400$ MLT, with increased response times on the nightside. For the 12-h sector centred on 1400 MLT, the weighted average response delay was found to be $1.3 \pm 0.8 \mathrm{~min}$, while for the 12-h sector centred on 0200 MLT the weighted average delay was found to increase to $8.8 \pm 1.7 \mathrm{~min}$. In the second method we first inspected the IMF data for sharp and enduring (at least $\sim 5 \mathrm{~min}$ ) changes in polarity of the north-south component, and then examined concurrent EISCAT flow data to determine the onset time of the corresponding enhancement or decay of the flow. For the case in which the flow response was timed from whichever of the flow components responded first, minimum response delays were again found at $\sim 1400$ MLT, with average delays of $4.8 \pm 0.5 \mathrm{~min}$ for the 12-h sector centred on $1400 \mathrm{MLT}$, increasing to
\end{abstract}

Correspondence to: $\mathrm{H}$. Khan

E-mail: hina.khan@ion.le.ac.uk
$9.2 \pm 0.8 \mathrm{~min}$ on the nightside. The response delay is thus found to be reasonably small at all local times, but typically $\sim 6$ min longer on the nightside compared with the dayside. In order to make an estimate of the ionospheric information propagation speed implied by these results, we have fitted a simple theoretical curve to the delay data which assumes that information concerning the excitation and decay of flow propagates with constant speed away from some point on the equatorward edge of the dayside open-closed field line boundary, taken to lie at $77^{\circ}$ magnetic latitude. For the combined cross-correlation results the best-fit epicentre of information propagation was found to be at 1400 MLT, with an information propagation phase speed of $9.0 \mathrm{~km} \mathrm{~s}^{-1}$. For the combined event analysis, the best-fit epicentre was also found to be located at 1400 MLT, with a phase speed of $6.8 \mathrm{~km} \mathrm{~s}^{-1}$.

Key words. Interplanetary physics (interplanetary magnetic fields) - Magnetospheric physics (Plasma convection; solar wind - magnetosphere interactions)

\section{Introduction}

Observations of plasma convection in the high-latitude ionosphere by polar-orbiting satellites have demonstrated that the form and magnitude of the flow depends on the direction and strength of the interplanetary magnetic field (IMF) which impinges on the dayside magnetopause boundary of the Earth's magnetosphere (e.g. Heppner, 1972; Reiff et al., 1981; Reiff and Burch, 1985). Figure 1 shows recent results derived by Weimer (1995) from electric field measurements made by the Dynamics Explorer 2 satellite. In this study electric potential patterns for various IMF conditions were obtained by fitting the data to a spherical harmonic expansion. The figure shows representative results where the IMF has been divided into eight $45^{\circ}$ sectors in the 


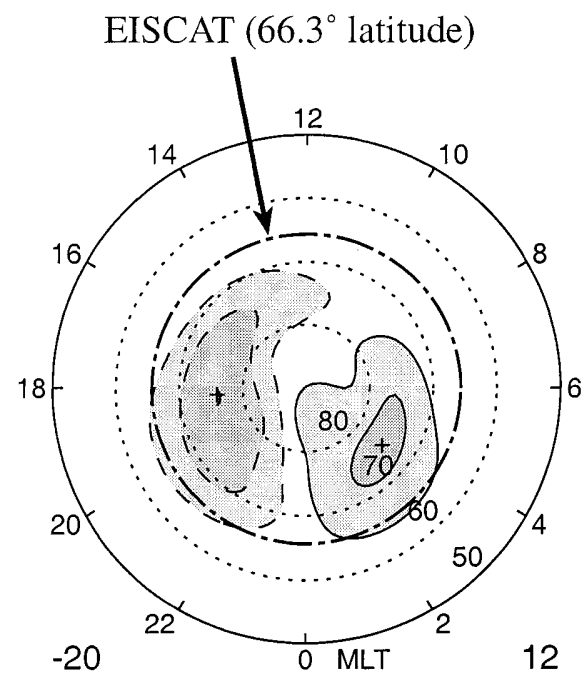

\section{Electric Potential}

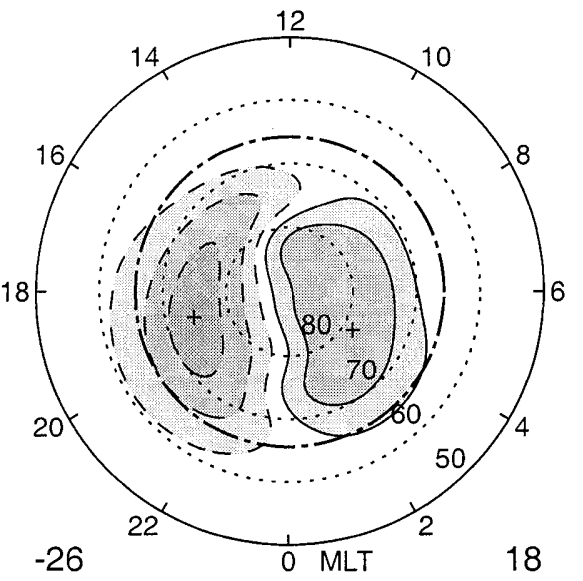

$-21$

$$
3.5<\mathrm{B}_{\mathrm{T}}<5.2 \mathrm{nT}
$$
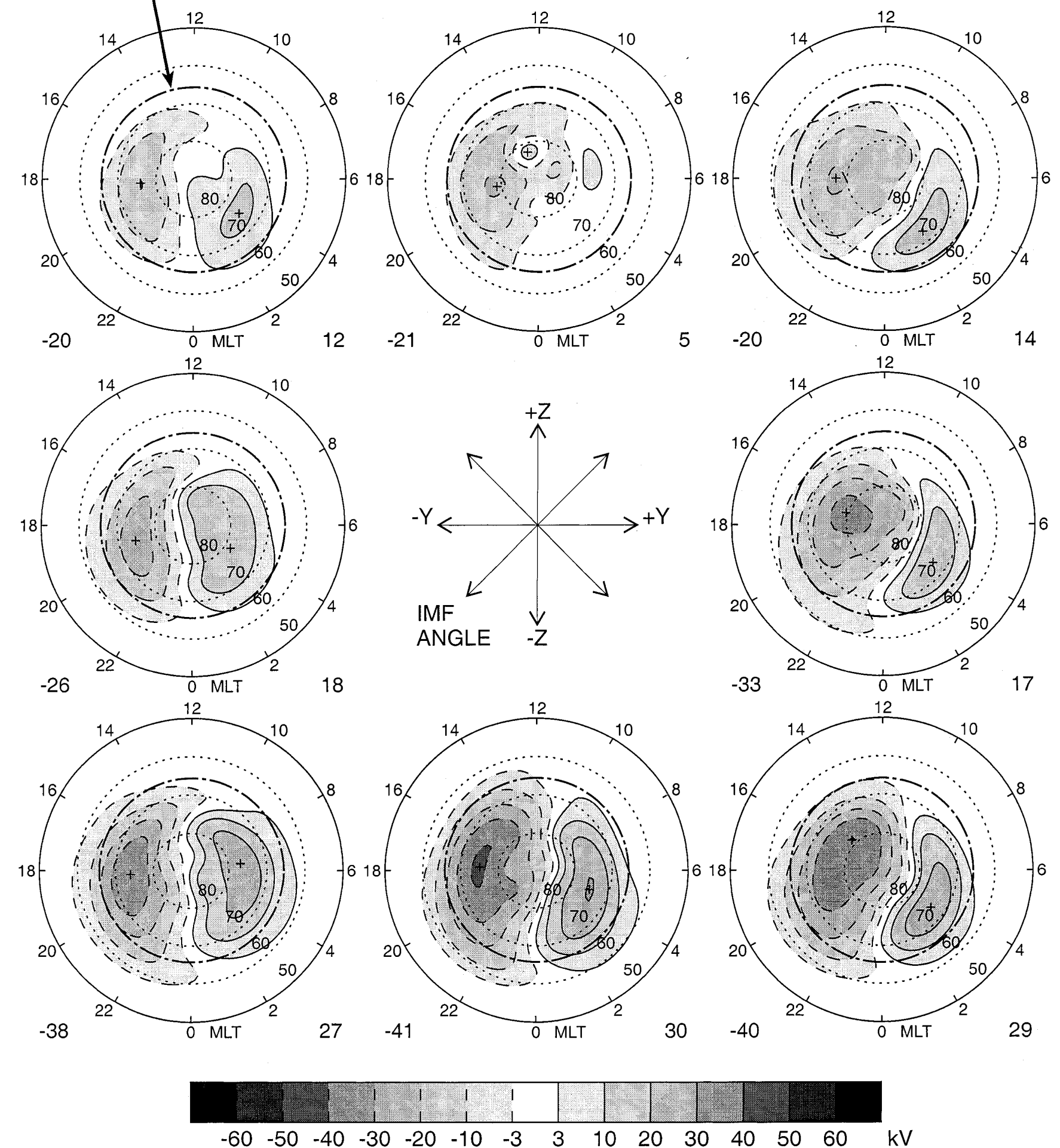

Fig. 1. Ionospheric potential contour plots (plasma streamlines) for eight $45^{\circ}$ intervals of the IMF vector in the GSM Y-Z plane, and where the total field strength in this plane $\left(\mathrm{B}_{\mathrm{T}}\right)$ lies between 3.5 and $5.2 \mathrm{nT}$. Positive and negative potentials are indicated by solid and

GSM Y-Z plane, in the case where the strength of the field in this plane $\left(\mathrm{B}_{\mathrm{T}}\right)$ lay between 3.5 and $5.2 \mathrm{nT}$. These patterns exhibit (a) a growth in the spatial size and dashed lines, respectively. The EISCAT radar location at $66.3^{\circ}$ magnetic latitude is marked by the dotted-dashed circle, (after Weimer, 1995)

transpolar voltage associated with the usual twin-cell flow as the IMF rotates from north to south; (b) highlatitude dawn-dusk flow asymmetries associated with 
the Y component of the IMF; and (c) evidence of additional dayside cells when the IMF points nearly directly northwards. Overall, such results provide convincing evidence that the primary process which couples solar wind momentum to the Earth's magnetosphereionosphere system is magnetic reconnection at the magnetopause (Dungey, 1961; Cowley, 1981; Lockwood, 1995).

Although the studies cited define how the highlatitude flow relates to the direction of the IMF, they provide little evidence about the time scale on which the flow reconfigures when the direction changes. The earliest information on this topic came from analyses of the response of ground magnetic disturbances to the IMF. Using globally distributed magnetic records, Nishida (1968) found that the DP 2 magnetic disturbance was excited with a $7 \pm 1$ min delay at the pole and a $9 \pm 5$ min delay near the equator following the arrival of southward-directed IMF at the bow shock. The equivalent DP 2 current system is of twin-vortical form and is believed to represent the magnetic counterpart of the twin-vortex flow shown in Fig. 1. Since the onward time for changes in the IMF to propagate from the bow shock to the dayside ionosphere is comparable to the delay measured by Nishida (1968), the implication is that the ionospheric flow response is very prompt indeed. Subsequent studies of magnetic disturbance considered the correlations between various interplanetary parameters, such as the "half-wave rectifier function" of Burton et al. (1975) or the "epsilon parameter" of Perreault and Akasofu (1978), and geomagnetic indices such as the auroral electrojet indices (Baker et al., 1981, 1983; Clauer et al., 1981; Bargatze et al., 1985). These studies indicated that two separate response times were present, with delays of 20 and $60 \mathrm{~min}$ relative to interplanetary conditions mapped from an upstream monitor to the subsolar magnetopause (though in making these estimates no account was taken either of the slowing of the flow in the magnetosheath, or of the subsequent Alfvénic propagation to the ionosphere, which will shorten these times by $\sim 10 \mathrm{~min}$ ). The shorter response delay was suggested to be associated with activity driven directly by solar wind coupling at the magnetopause, while the longer time scale was suggested to relate to eventual substorm activity on the nightside. Time scales of $\sim 20-30 \mathrm{~min}$ for the reconfiguration of the twin-vortex flow as a whole following southward turns of the IMF have also been reported by Hairston and Heelis (1995) using ion flow measurements from DMSP spacecraft.

Ground-based radars have also been used to study the ionospheric flow, and provide an ideal means of monitoring its temporal variations in a given region. By combining data from the EISCAT UHF radar with IMF measurements made by the AMPTE-UKS and -IRM spacecraft, Etemadi et al. (1988) and Todd et al. (1988) determined the response delay of the flow at $71^{\circ}-73^{\circ}$ magnetic latitude to variations in the northsouth component of the IMF mapped to the subsolar magnetopause. Observations were confined mainly to the dayside ionosphere (08-19 MLT), and showed minimum delays of $\sim 5 \mathrm{~min}$ in the early afternoon sector, increasing to $\sim 10$ min towards dawn and dusk. These estimates should be reduced by $\sim 2 \mathrm{~min}$ if the Alfvénic propagation time from the subsolar magnetopause to the ionosphere is taken into account. Similar very short response delays on the dayside have also been reported in HF radar data by Ruohoniemi et al. (1993). The increasing delays away from noon imply that the region of enhanced flow expands rapidly away from the region of the dayside cusp to earlier and later local times, and also, presumably, over the polar cap into the nightside. The dayside azimuthal phase speed at $71^{\circ}-73^{\circ}$ implied by the results of Etemadi et al. (1988) and Todd et al. (1988) is $5-10 \mathrm{~km} \mathrm{~s}^{-1}$. Direct measurements of the dayside phase speed at similar latitudes have also been made using both radar data and ground-based magnetic records. Lockwood et al. (1986) and Lockwood et al. (1993) report values of 2.6 and $6 \mathrm{~km} \mathrm{~s}^{-1}$, respectively, using EISCAT UHF and VHF radar data, while Saunders et al. (1992) derived a value of $5 \mathrm{~km} \mathrm{~s}^{-1}$ from CANOPUS magnetometer network data. Based on these observations, Cowley and Lockwood (1992, 1997) have suggested a theoretical picture in which flow changes occurring in response to variations in the direction of the IMF begin in the dayside cusp near noon, and propagate away from this vicinity to establish a new steady-state convection pattern over intervals of 10-15 min. This expansion of the flow pattern corresponds to the ionospheric image of the expansion of the perturbed flow region at large distances in the magnetosphere. For example, following a north-to-south turning of the IMF and the onset of open flux production at the dayside magnetopause, the perturbed region will initially be confined to the dayside where the open flux tubes are produced, followed by expansion into the nightside as the open tubes are swept antisunward by the magnetosheath flow and the disturbance propagates into the tail.

On the basis of this we may thus expect that the component of the nightside flow which responds directly to the IMF should appear with a response delay of $\sim 10$ min relative to first effects in the cusp, this delay corresponding to an expansion phase speed of $\sim 5 \mathrm{~km} \mathrm{~s}^{-1}$. To date, however, there have been very few direct determinations of the nightside delay. Estimates from radar data obtained in a few case studies vary from $\sim 10$ to $\sim 40 \mathrm{~min}$ (Lester et al., 1993; Taylor et al., 1994). However, Ruohoniemi and Greenwald (1998) have recently presented an example using SuperDARN HF radar data in which changes in flow occur in apparent response to a southward turn of the IMF which are essentially simultaneous (within $\sim 2 \mathrm{~min}$ ) on both the day and night sides at $\sim 75^{\circ}-80^{\circ}$ latitude. In addition, Ridley et al. $(1998,1999)$ have interpreted flow patterns obtained using the AMIE modelling technique as indicating a coherent evolution of the flow pattern over the whole polar ionosphere with no significant delays greater than $\sim 1$ min between day and night sides. They determined that the flow change begins $\sim 8 \pm 8 \mathrm{~min}$ after the corresponding IMF change arrives at the subsolar magnetopause. However, the 
interpretation of their data has been disputed by Lockwood and Cowley (1999).

In this study our principal aim is to examine the response time of the ionospheric flow to the north-south component of the IMF, and to determine its local time dependence. To this end we have used a large database of measurements of the ionospheric flow observed overhead at Tromsø by the EISCAT incoherent scatter radar, and have combined these with simultaneous measurements of the interplanetary parameters measured upstream of the Earth by the IMP-8 spacecraft. The next section provides details of the instrumentation and database, while cross-correlation and event analyses follow in Sects. 3 and 4.

\section{Instrumentation and data sets}

\subsection{The EISCAT CP-1-K experiment}

The ionospheric flow measurements employed in this study were obtained by the tristatic EISCAT UHF incoherent scatter radar system using the CP-1-K common programme experiment, which has been run typically on several days each year since 1993. In this experiment the radar beam is pointed along the magnetic field line at Tromsø (Norway), approximately southward at an elevation of $77.5^{\circ}$, while the remote site receivers at Kiruna (Sweden) and Sodankylä (Finland) are pointed at a fixed intersection with the transmitter beam at an F-region altitude of $278 \mathrm{~km}$. This arrangement allows continuous tristatic measurement of the plasma flow at a single point essentially overhead at Tromsø. The data are integrated over $2 \mathrm{~min}$ intervals, which sets the limit on the time resolution of this study.

The CP-1-K tristatic observing point corresponds to a magnetic latitude of $66.3^{\circ}$. This is a somewhat lower latitude than those $\left(\sim 71^{\circ}-73^{\circ}\right)$ investigated in the previous studies by Todd et al. (1988) and Etemadi et al. (1988), which used beam-swung EISCAT data from the poleward-pointing "Polar" experiment. Comparison with the flow maps in Fig. 1 shows that on the dayside $66.3^{\circ}$ typically lies equatorward of the flow cells for northward IMF, but just within their equatorward border for southward IMF. On the nightside the EISCAT latitude lies on average in the equatorward border of the flow cells for northward IMF, and well within the cells on closed field lines for southward IMF. Thus the response of the flow to north-south changes in the IMF should be observable in these data at all local times, a conclusion which is borne out by the data themselves, as we will see later. The response time of the flow expected on the basis of previous results varies considerably between the prompt, few minute, responses observed on the dayside by Etemadi et al. (1988), Todd et al. (1988), and Ruohoniemi et al. (1993), and the more extended intervals of 20-30 min which are probably more characteristic of reconfigurations of the flow pattern as a whole reported by Hairston and Heelis (1995). On the nightside, Ridley et al. (1998) and Ruohoniemi and Greenwald (1998) indicate flow re- sponses that are essentially simultaneous with the dayside flow, within 1-2 min or less, while other observations cited suggest a slower propagation. If, for example, flow changes propagate away from the openclosed field line boundary at noon at phase speeds e.g. of $\sim 5-10 \mathrm{~km} \mathrm{~s}^{-1}$, the resulting delay at EISCAT should vary between $\sim 2-4 \mathrm{~min}$ at noon and $\sim 7-14 \mathrm{~min}$ at midnight. The 2-min averaged flow data employed here should thus be capable of distinguishing between these possibilities and of resolving day-night differences of this order, though only just so for phase speeds at the top end of the range considered likely.

\subsection{IMP-8 interplanetary data}

For purposes of this study the EISCAT radar data have been combined with observations of the IMF made upstream of the bow shock by the IMP-8 spacecraft. This spacecraft moves in a near-circular $35 \mathrm{R}_{\mathrm{E}}$ radius orbit, and provides IMF vector data at $15 \mathrm{~s}$ resolution and solar wind plasma parameters with a resolution between 60 and $300 \mathrm{~s}$. Since the flow response delays which we wish to measure lie typically in the range $\sim 5-20 \mathrm{~min}$, as indicated, it is crucial to make a careful estimate of the propagation delay between fields observed at the spacecraft and the arrival of their first effects in the dayside ionosphere. Full details are provided in the Appendix, but, briefly, the propagation delay consists of three components. The first is the propagation time in the solar wind between arrival at the spacecraft and arrival at the subsolar bow shock. This has been calculated by using sharp changes in the IMF observed during particular intervals to determine the orientation of the IMF "phase fronts" in the interplanetary medium, and hence the distance along the Earth-Sun line to the subsolar bow shock of the phase front which passes through the spacecraft. The latter distance is then divided by the observed velocity of the solar wind to give the propagation time to the subsolar shock. This procedure is generally applied to several field discontinuities during a particular interval, from which a value is chosen which is representative of the interval as a whole. The second element is the frozen-in transit time across the subsolar magnetosheath. To estimate this we require models of the location of the subsolar bow shock and magnetopause, and the variation of the plasma speed between them. The shock and magnetopause locations have been derived from the comprehensive empirical studies by Peredo et al. (1995) and Roelof and Sibeck (1993), respectively, and we take a linear variation of the subsolar flow from the value just downstream from the shock, determined from typical shock jump conditions, and a nominal $20 \mathrm{~km} \mathrm{~s}^{-1}$ reconnection-associated inflow at the magnetopause. The third element is the Alfvénic propagation of the effects of magnetopause processes along newly-opened field lines to the cusp ionosphere. A constant propagation time of $2 \mathrm{~min}$ has been assumed, derived from the typical periods of fundamental mode eigenoscillations of the high-latitude field lines. 
Using these procedures, an IMF delay time representative of 2-h intervals of radar data has been determined. Typical values lie in the range 5-15 min, with an estimated random uncertainty of about $\pm 2 \mathrm{~min}$ and an estimated systematic uncertainty which is of the same order or less (see Appendix). This estimated propagation delay is removed prior to averaging the interplanetary data over the "same" 2 min intervals as the radar data. These joint 2 min-averaged data sets form the basis of the cross-correlation analyses presented in Sect. 3. The IMF propagation delays for the event analysis in Sect. 4 were determined in a similar manner, except that the "phase front" for each IMF event was determined and employed individually, and the solar wind parameters were averaged locally in its vicinity (see Appendix).

\subsection{The combined data set}

The database of EISCAT CP-1-K flow data and IMP-8 solar wind/IMF data employed in this study, obtained during the interval 1993-97, total about 300 simultaneous hours. The data span all local times, though the hemisphere centred on dusk is favoured relative to the hemisphere centred on dawn. Typically $\sim 8$ experimentdays contribute data in each local time interval in the dawn hemisphere, compared with $\sim 13$ experiment-days in the dusk hemisphere. An example of the data is presented in Fig. 2, where we show a 2-h interval spanning 0900-1100 UT on 14 February, 1996, corresponding to $1130-1330$ MLT in the noon sector. The top panel of the figure shows IMF $B_{z}$, while the centre and lower panels show the field-perpendicular ionospheric flow velocity components, $v_{N}$ positive towards magnetic north and $v_{E}$ positive towards magnetic east. The results of Weimer (1995) reproduced in Fig. 1 indicate that the flow should be small for IMF $B_{z}$ positive, while westward and northward ("dusk cell") flows should appear for IMF $B_{z}$ negative. Correspondingly, at the start of the interval when IMF $B_{z}$ was positive, the observed flow scatters about zero. However, two sharp southward turns of the IMF occurred, observed at IMP-8 at 0925 and 1019 UT, which are marked by the vertical dashed lines in the upper panel. The first effects in the ionosphere, determined from the individual orientations of the field discontinuities concerned as indicated, are then expected at 0938 and 1032 UT (the delay being $13 \mathrm{~min}$ in both cases), as indicated by the vertical dashed lines in the lower two panels. It can be seen that both southward turns resulted in the excitation of $\sim 200-300 \mathrm{~m} \mathrm{~s}^{-1}$ westward flows and less distinct poleward flows, as expected, with first changes occurring at about 0941 and 1035 UT respectively, as marked by the vertical dashed lines in the lower panel. The response delays are thus about $3 \mathrm{~min}$ in each case, within an uncertainty band that is $\sim 2 \mathrm{~min}$. The flow response is thus very prompt near noon, in conformity with the previous results e.g. of Etemadi et al. (1988) and Todd et al. (1988). These authors reported a $\sim 5$ min response delay in this local time sector, but they did not subtract the $\sim 2$ min magnetopause-toionosphere propagation delay in the IMF signal which we have included here.

Having now discussed the nature of the data, in the next section we will present the results of a crosscorrelation analysis between the ionospheric flow and interplanetary parameters, which seeks to determine the flow response time scale at all local times. This study will

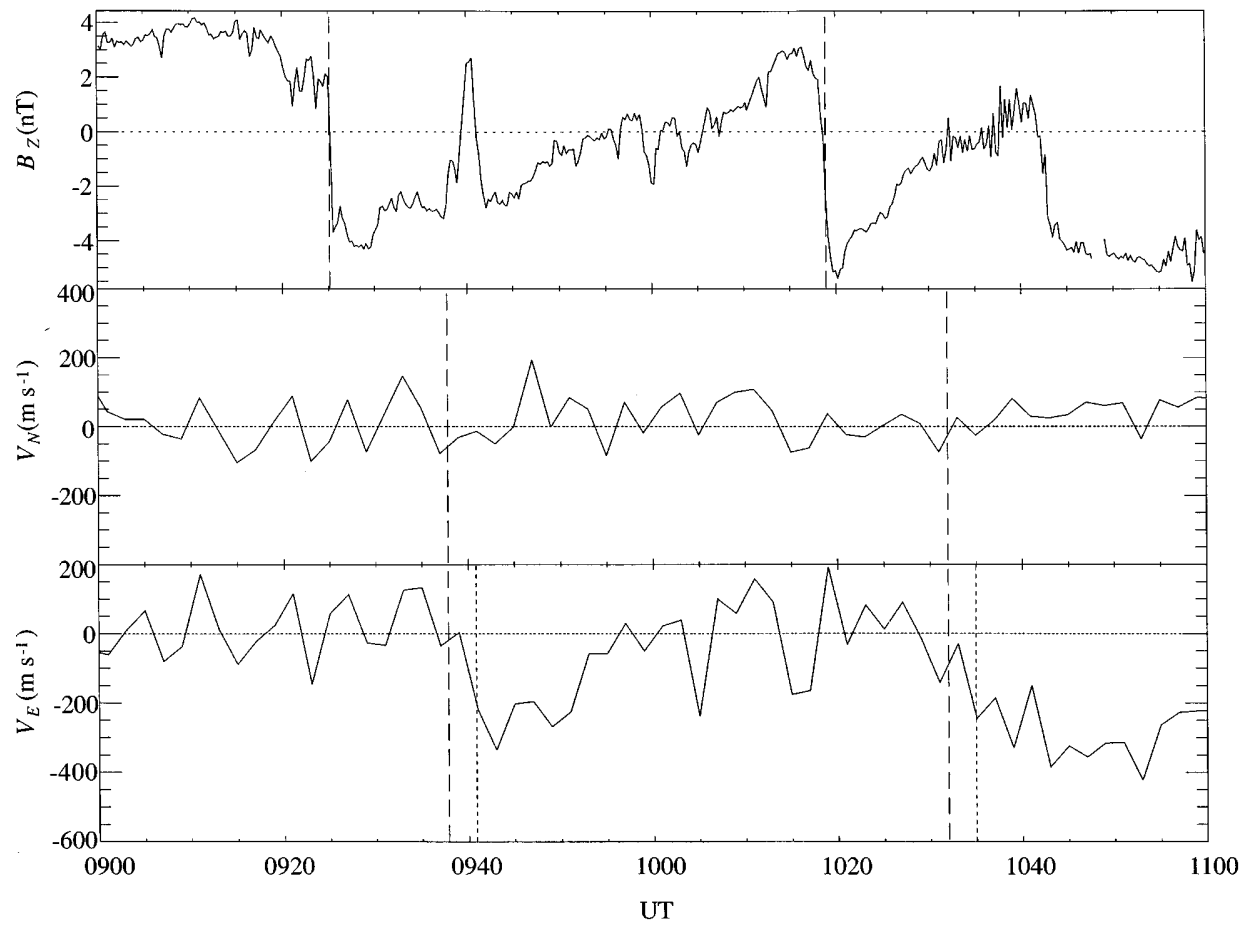

Fig. 2. Simultaneous IMP-8 magnetic field data and EISCAT CP-1-K velocity data for the interval 0900 1100 UT (1130-1330 MLT) on 14 February, 1996. The top panel shows $15 \mathrm{~s}$ values of the IMF $B_{z}$ field component, while the lower two panels show 2 min values of the magnetic north and magnetic east components of the field-perpendicular velocity measured by the radar. Two southward turnings of the IMF are marked by the dashed vertical lines in the upper panel, whose effects are expected to arrive in the ionosphere at the times indicated by the dashed vertical lines in the lower panels. The times at which the first clear effects were observed in the flow components are shown by the dotted vertical lines in the lower panels 
then be supplemented in Sect. 4 with an analysis of individual events such as those illustrated here.

\section{Cross-correlation analysis}

We describe the results of a cross-correlation analysis between the 2 min-averaged EISCAT velocity data and the similarly-averaged IMP-8 interplanetary data from which the propagation delay to the cusp ionosphere has been removed, as described above. The radar data were divided into overlapping 2 -h intervals of MLT $(\mathrm{MLT} \approx \mathrm{UT}+2.5 \mathrm{~h})$, and cross-correlations performed on both eastward and northward flow components separately. The interplanetary parameter chosen for this analysis was the "half-wave rectifier" function $V^{2} B_{S}$, where $V$ is the solar wind speed, and $B_{S}=B_{z}$ when the latter is negative, while $B_{s}=0$ when $B_{z}$ is positive. In common with the results of the previous cross-correlation study by Etemadi et al. (1988), initial investigation indicated that this parameter produces correlation coefficients which are generally as high or higher than other simple related interplanetary parameters. In particular it produces higher correlations than related functions that use $B_{z}$ directly, reflecting the fact that at the latitude of Tromsø the flow is unresponsive to the IMF (and is generally small) when $B_{z}$ is positive, but grows in magnitude when $B_{z}$ is negative, as we will demonstrate in the results presented below.

For each 2-h MLT interval of radar data the crosscorrelation coefficients of $v_{E}$ and $v_{N}$ with $V^{2} B_{S}$ were calculated versus time lag over an interval of $\pm 120 \mathrm{~min}$, with a resolution of $2 \mathrm{~min}$ (the resolution of the data sets). For positive lags the interplanetary medium leads the ionosphere, while for negative lags the ionosphere (unphysically) leads the interplanetary medium. Before presenting the results, however, it is appropriate to comment on the interpretation of the resulting crosscorrelograms, particularly since it seems clear that the high-latitude flow varies in response to the IMF on more than one time scale. The first time scale is the delay time of the excitation and decay of the flow in response to north-south changes in the IMF, whose nature is demonstrated in Fig. 2 and in the previous studies presented e.g. by Todd et al. (1988). All previous evidence indicates that the delay times involved in this process are of order a few minutes relative to the arrival of first effects, certainly in the dayside ionosphere. The second relates to changes in the local flow which are due to overall expansions or contractions in the flow pattern as the amount of open flux in the system changes. Such changes take place on a significantly longer time scale. For example, unbalanced dayside reconnection at rate of $100 \mathrm{kWb} \mathrm{s}^{-1}$ (corresponding to a transpolar voltage of $100 \mathrm{kV}$ ) will result in a uniform equatorward expansion of the open-closed field line boundary at a speed of $\sim 200 \mathrm{~m} \mathrm{~s}^{-1}$. The time scale required for a given point in the ionosphere to move significantly relative to the flow pattern, corresponding to a boundary motion of a few degrees of latitude, for example, is then of order several tens of minutes (about ten minutes per degree). We may therefore envisage that as the IMF switches between north and south polarities the ionospheric flow will be similarly modulated with the short delay time scale, whilst also undergoing variations on the longer time scale during each southward-directed IMF interval.

We may then ask at what delay time the peak in the cross-correlation coefficient will occur? The answer to this question depends upon the nature of the flow variation which is produced by the variation in the size of the flow cells on the longer time scales. If the change in the flow component relative to the (usually small) positive IMF $B_{z}$ background has a consistent sense (e.g. a consistently westward or eastward flow) whose absolute magnitude may be modulated by the size of the flow cells, then it is easy to show that an extremum in the cross-correlation coefficient will occur at the lag corresponding to the flow excitation and decay delay time, irrespective of the nature of the long time-scale modulation. This can be seen from the fact that a maximum "overlap" will invariably occur between the enhanced flow component and the IMF input function at this lag, such that a small shift in lag in either direction will reduce the "overlap" and hence the cross-correlation coefficient. Figure 1 shows that this is the situation which will prevail at EISCAT latitudes in most local time sectors, since the radar will usually be located in the equatorward region of the flow cells where the flows will generally be consistently westward in the dusk cell and consistently eastward in the dawn cell. However, there is one local time sector where this general conclusion does not apply, namely the pre-midnight Harang region. It can be seen from Fig. 1 that in this region the flows will generally be westward for positive and small IMF $B_{z}$, while reversing to eastward for significantly negative IMF $B_{z}$. Typically then, if the direction of the IMF reverses from north to south, we may expect that the initial flow excitation will be in the westward direction, reversing later to eastward on a time scale (a few tens of minutes) which may be dependent on the strength of the southward field. In this case consideration of the "overlap" between the flow component and the interplanetary input shows that a peak in the cross-correlation coefficient need not occur at the lag corresponding to the flow excitation and decay delay time as above, but may occur at a later time characteristic of the reversal in the sense of the flow relative to the positive IMF $B_{z}$ "background" value.

These qualitative conclusions are illustrated quantitatively in Fig. 3. We have constructed synthetic data sets consisting of 1000 simultaneous "IMF $B_{s}$ " and "ionospheric velocity" data points, corresponding to $33.3 \mathrm{~h}$ of the 2-min averaged data employed here. A random number generator has then been used to select the times of $N_{I M F}$ switches in the value of "IMF $B_{s}$ ", between zero, corresponding to northward IMF, and $-1 \mathrm{nT}$, corresponding to southward IMF, or vice versa. A typical example is shown in the upper panel of Fig. 3a, where $N_{I M F}=75$, such that the average time between "IMF" switches is $\sim 26 \mathrm{~min}$, corresponding to $\sim 13$ data points. Two synthetic "velocity" data sets are shown beneath. In the middle panel the velocity is taken 
to be a random number between 0 and $-300 \mathrm{~m} \mathrm{~s}^{-1}$ whenever "IMF $B_{s}$ " is negative, and to be zero when "IMF $B_{s}$ " is zero. This corresponds to the excitation of a flow of variable magnitude, but consistent sense, in concert with a southward IMF. The cross-correlation function between these data sets is shown in the upper panel of Fig. 3b, computed every two minutes over a range of lags of $\pm 120 \mathrm{~min}$, with positive lags indicating that the "IMF" leads the "velocity", as indicated. A single clear peak is obtained at zero lag, as anticipated in the discussion. The same result is obtained for all the synthetic data sets of this nature which we have generated, having different random variations in the times of the IMF changes and in the velocity, and for various values of $N_{I M F}$ (between 50 and 100), as must be the case. In the synthetic "velocity" data shown in the bottom panel of Fig. 3a, however, the flow is switched "on" and "off" in concert with the "IMF" function just as before, but now the flow may take positive or negative values. Specifically, the flow is taken to be negative (at a random value between 0 and $-300 \mathrm{~m} \mathrm{~s}^{-1}$ ) for 10 successive "flow on" data points (i.e. $20 \mathrm{~min}$ of flow), after which it becomes positive (at a random value between 0 and $+300 \mathrm{~m} \mathrm{~s}^{-1}$ ) for all subsequent "flow on" data points, until such time as a "substorm" takes place.
After this time the flow reverts to negative for 10 successive points, followed by positive again until the next "substorm", etc. The times of $N_{S}$ "substorms" are again chosen randomly, and are indicated in the lower panel of Fig. 3a by the arrows, where we have chosen $N_{S}=15$ for the data set displayed (i.e. an average time of $\sim 2 \mathrm{~h}$ between "substorms"). This algorithm is intended in the simplest possible way to simulate conditions in the Harang region, where the flow direction may change from west to east after a few tens of minutes of southward IMF due to polar cap expansion (specifically $20 \mathrm{~min}$ in our algorithm), followed by the reappearance of westward flow when the polar cap subsequently contracts after a substorm. The cross-correlogram for this data set is shown in the lower panel of Fig. 3b. Here the cross-correlation values are lower than before, and peak at a lag of $20 \mathrm{~min}$, corresponding to the time scale of the switch in the sense of the flow. While such results are typical, we find that other randomly-chosen data sets having the same basic parameters (i.e. the same $N_{I M F}$ and $N_{S}$ values) can produce peaks at various lags between $\sim 0$ and $\sim 20 \mathrm{~min}$.

When examining the cross-correlation results, therefore, attention must be paid to the nature of the flow response to IMF $B_{z}$. If the flow response is in a

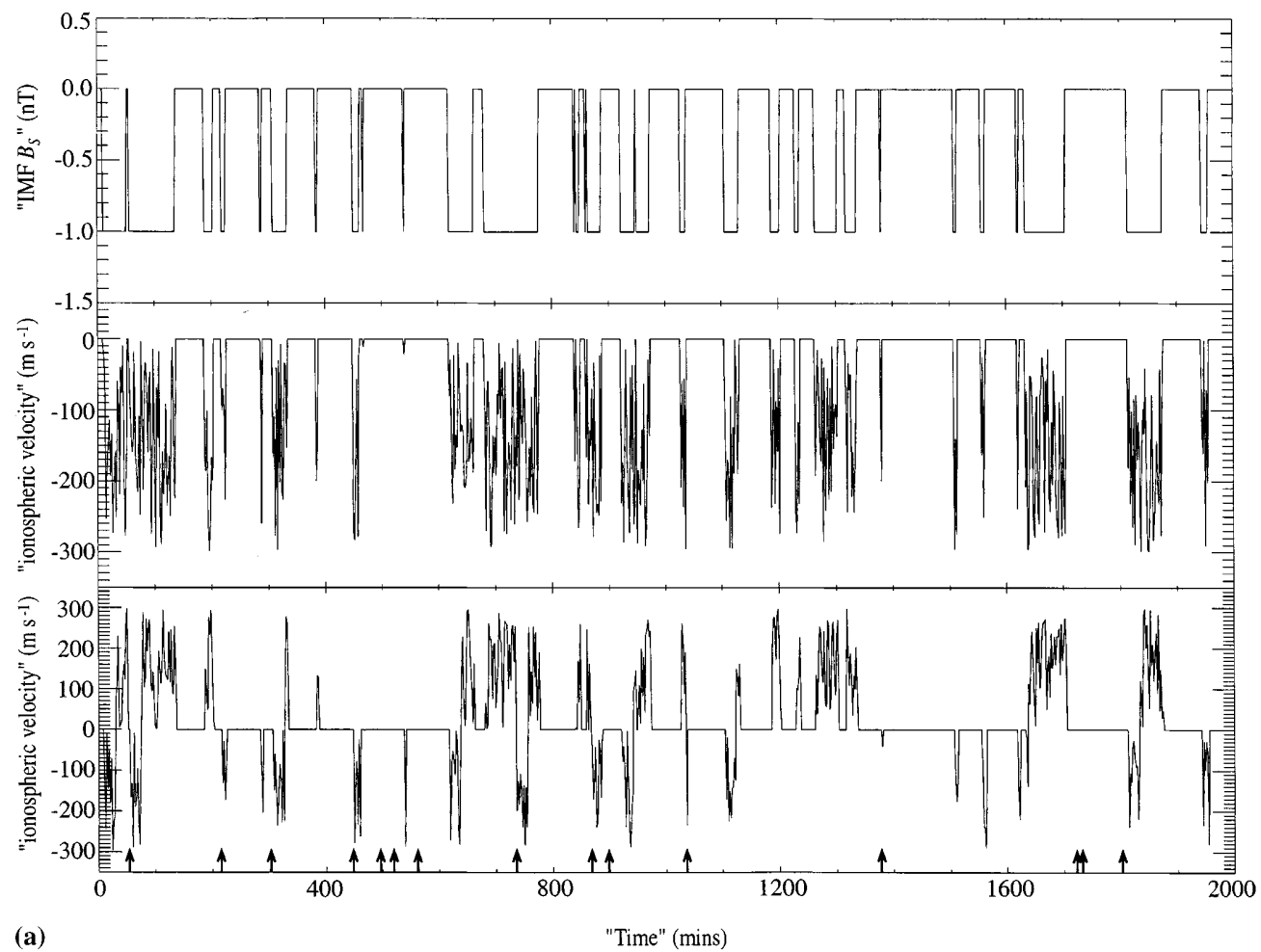

Fig. 3. a Synthetic data sets of 1000 2-min "IMF $B_{s}$ " values (upper panel) and simultaneous "ionospheric velocity" components determined from two different algorithms (lower two panels). In the upper panel, "IMF $B_{s}$ " is switched between zero and $-1 \mathrm{nT}$ (or vice versa) at 75 randomly chosen times. In the middle panel the "velocity" is switched "on" and "off" in concert with "IMF $B_{s}$ ", and is allowed to have a randomly-chosen negative value between 0 and $-300 \mathrm{~m} \mathrm{~s}^{-1}$ when "IMF $B_{s}$ " is $-1 \mathrm{nT}$, and is zero when "IMF $B_{s}$ " is zero. In the lower panel the "velocity" is switched "on" and "off" by "IMF $B_{s}$ " in

just the same way, but may now take positive as well as negative values. Specifically, the flow is initially negative for 10 successive "on" points (i.e. for $20 \mathrm{~min}$ ), and then switches to positive for all "on" points thereafter until a "substorm" takes place. After the "substorm" the flow reverts to negative for 10 successive "on" points, before switching to positive again for all subsequent "on" points until the next "substorm", etc. Fifteen "substorms" are taken to occur during the interval, at randomly-determined times shown by the arrows in the lower panel 
Cross correlation of model "IMF $B_{S}$ " and "ionospheric flow"

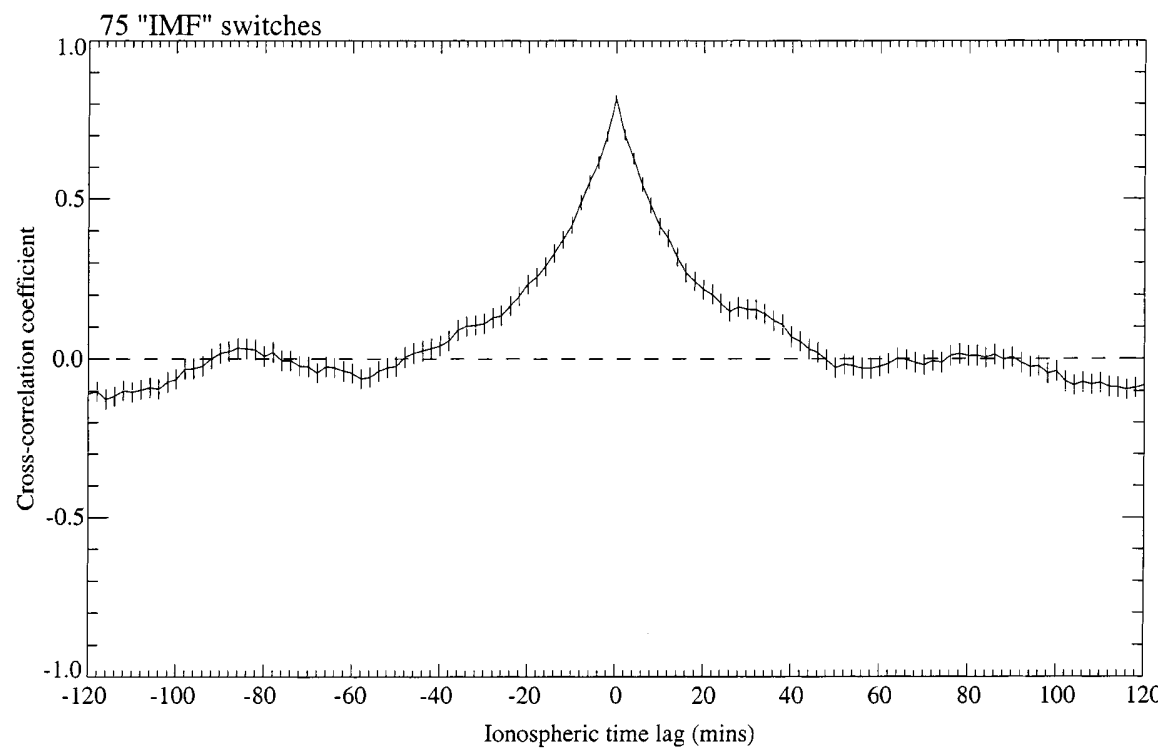

Cross correlation of model "IMF $B_{S}$ " and "ionospheric flow"

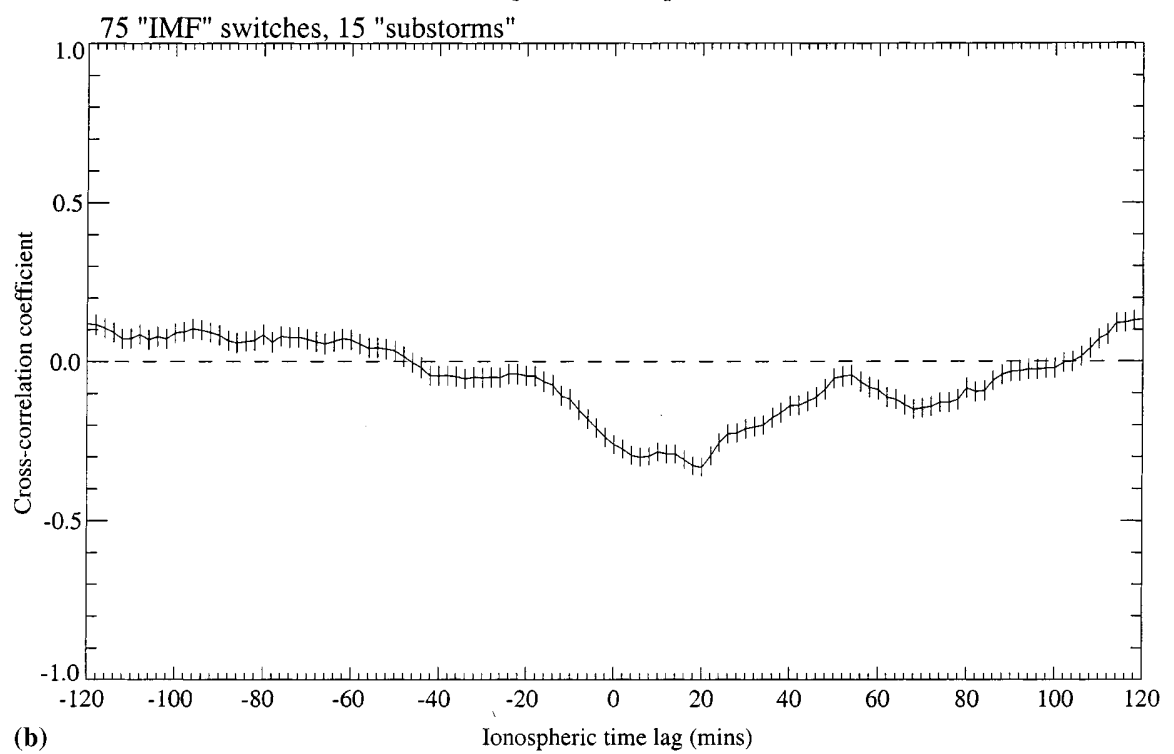

Fig. 3. b Cross-correlograms corresponding to the two synthetic "IMF"-"ionospheric velocity" data sets shown in a. The upper panel corresponds to the "velocity" data shown in the middle panel of a, while the lower panel corresponds to the "velocity" data shown in the lower panel of a consistent sense relative to the IMF $B_{z}$ positive "background" value (taken to be zero in the above synthetic data sets), then we may reasonably interpret the time lag of the peak correlation as the flow excitation and decay response delay. However, if the flow is found to reverse in sense with changing IMF $B_{z}$, relative to the $B_{z}$ positive "background", then the lag of the peak correlation need not correspond to the flow excitation and decay delay of primary interest here, but may occur at longer delays characteristic of the flow reversal time scale. We may further anticipate on the basis of Fig. 1 that such an effect is most likely to occur in the premidnight Harang region, where flows typically reverse from west to east as IMF $B_{z}$ switches from positive to negative. This effect needs to be borne in mind when considering the results presented.
Figure 4 thus presents representative results for various 2-h MLT intervals, where all the flow data available for the interval have been combined together to produce the results shown. In each panel results for $v_{E}$ are shown on the left, while those for $v_{N}$ are shown on the right. The upper graphs in each panel show the cross-correlation coefficient versus lag. The error bars show the standard error of the coefficient, given by $\varepsilon=\left(1-r^{2}\right) /(n-2)^{1 / 2}$, where $r$ is the value of the crosscorrelation coefficient and $n$ is the number of data values from which it was determined. This formula is valid when $n$ is large ( $n>100$, say), a condition well satisfied here where the number of 2-min data values in a given 2-h MLT interval varies from a minimum of about 200 to a maximum of about 700 (see Fig. 5). The 99.9\% significance level derived from Student's $t$-test is also shown by the dot-dash lines; cross-correlation coeffi- 
cients with magnitudes smaller than this are not considered statistically significant. The lower graphs in each panel then show scatter plots of the respective velocity components versus $V^{2} B_{z}$, derived using the lag corresponding to the peak in the cross-correlation curve as indicated at the top of each plot. The interplanetary parameter plotted is not $V^{2} B_{z}$ directly, but $\left(V\left(\mathrm{~km} \mathrm{~s}^{-1}\right) /\right.$ $500)^{2} B_{z}$, in units of $\mathrm{nT}$. That is, we have normalised the solar wind speed $V$ to a nominal value of $500 \mathrm{~km} \mathrm{~s}^{-1}$ so that the variation of the ionospheric flow with typical IMF $B_{z}$ values can more easily be appreciated. The solid lines in these plots represent the least-squares best fit to the half-wave rectified interplanetary function (i.e. a linear variation for IMF $B_{z}$ negative, and a constant value for IMF $B_{z}$ positive).

Figure $4 \mathrm{a}$ shows results for the 2-h interval centred on noon (1100-1300 MLT), containing 608 flow measurements. The cross-correlogram for $v_{E}$ shows a highly significant maximum near zero lag, with a peak coefficient of 0.50 at a lag of 2 min (which is thus in accord with the individual events shown in Fig. 2). The uncertainty in the determination of the lag at the peak
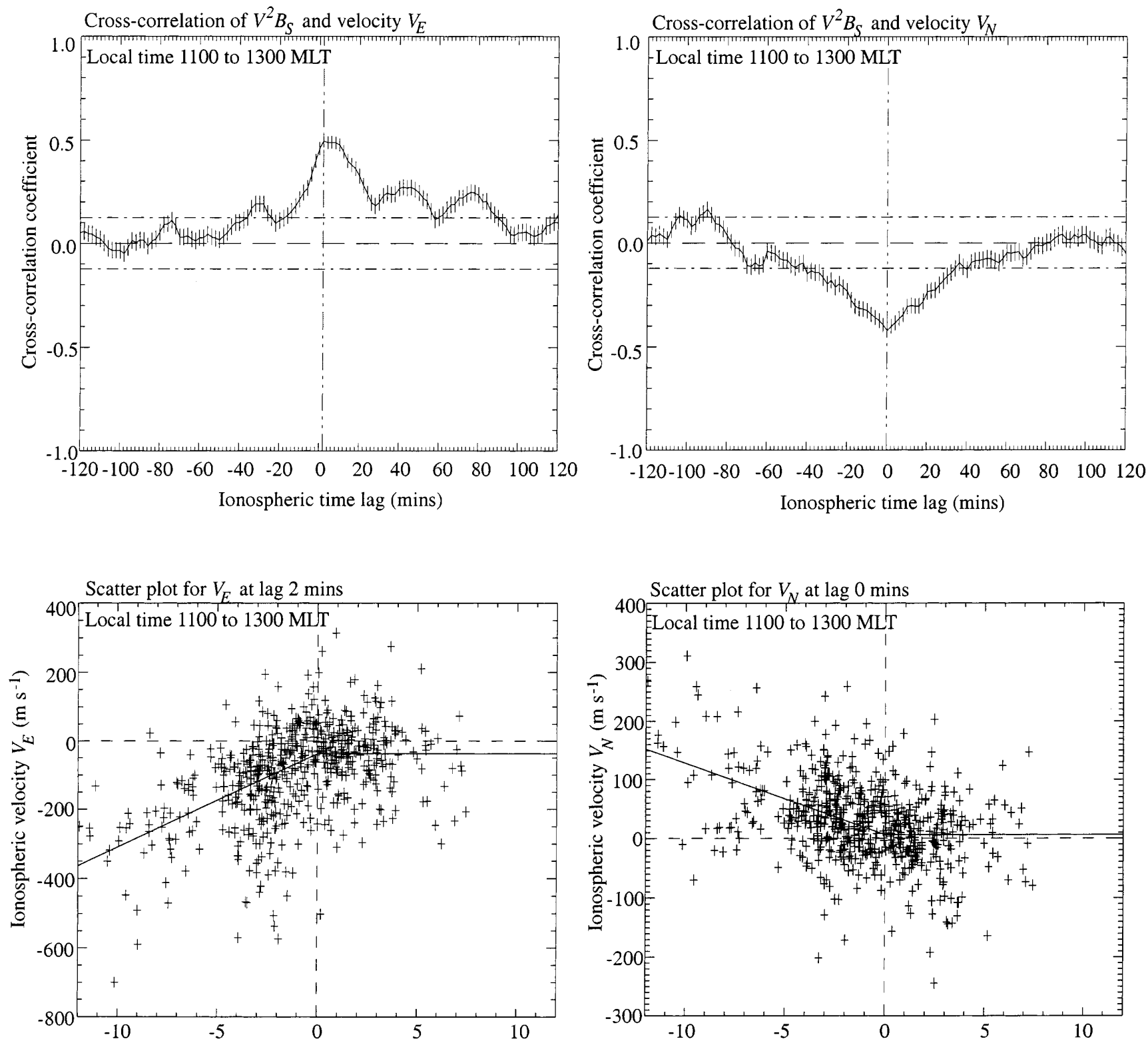

(a)

$\left(V\left(\mathrm{~km} \mathrm{~s}^{-1}\right) / 500\right)^{2} B_{Z}(\mathrm{nT})$

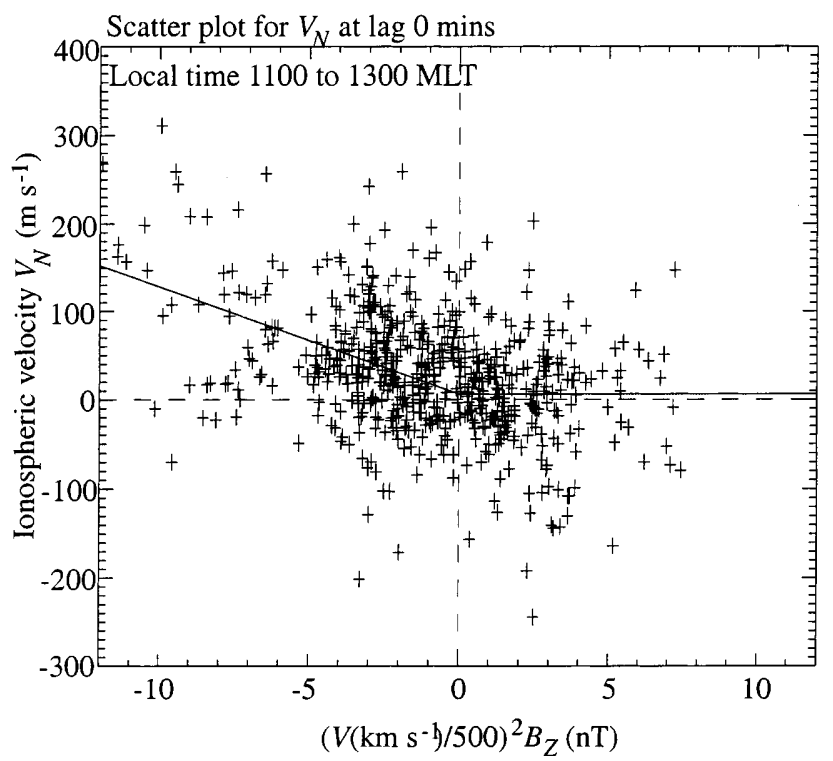

Fig. 4. a Results of the cross-correlation analysis between $V^{2} B_{s}$ and the eastward (left hand) and northward (right hand) flow components for the 2-h interval of local time centred on 1200 MLT. The upper panels show the value of the cross-correlation coefficient versus lag in the interval $\pm 120 \mathrm{~min}$ at $2 \mathrm{~min}$ resolution, with positive lags representing the physical scenario in which the interplanetary medium leads the ionosphere. The nearly horizontal dot-dashed lines show the

$99.9 \%$ significance level calculated by the Student's $t$-test. The vertical dot-dashed line marks the peak in the cross-correlogram. The lower panels provide a corresponding scatter plot of the flow component versus $\left(V\left(\mathrm{~km} \mathrm{~s}^{-1}\right) / 500\right)^{2} B_{z}$ in $\mathrm{nT}$, plotted at the lag of the peak correlation coefficient shown in the plot above. A linear least-squares fit of the ionospheric velocity data to the function $V^{2} B_{s}$ is indicated by the solid lines 

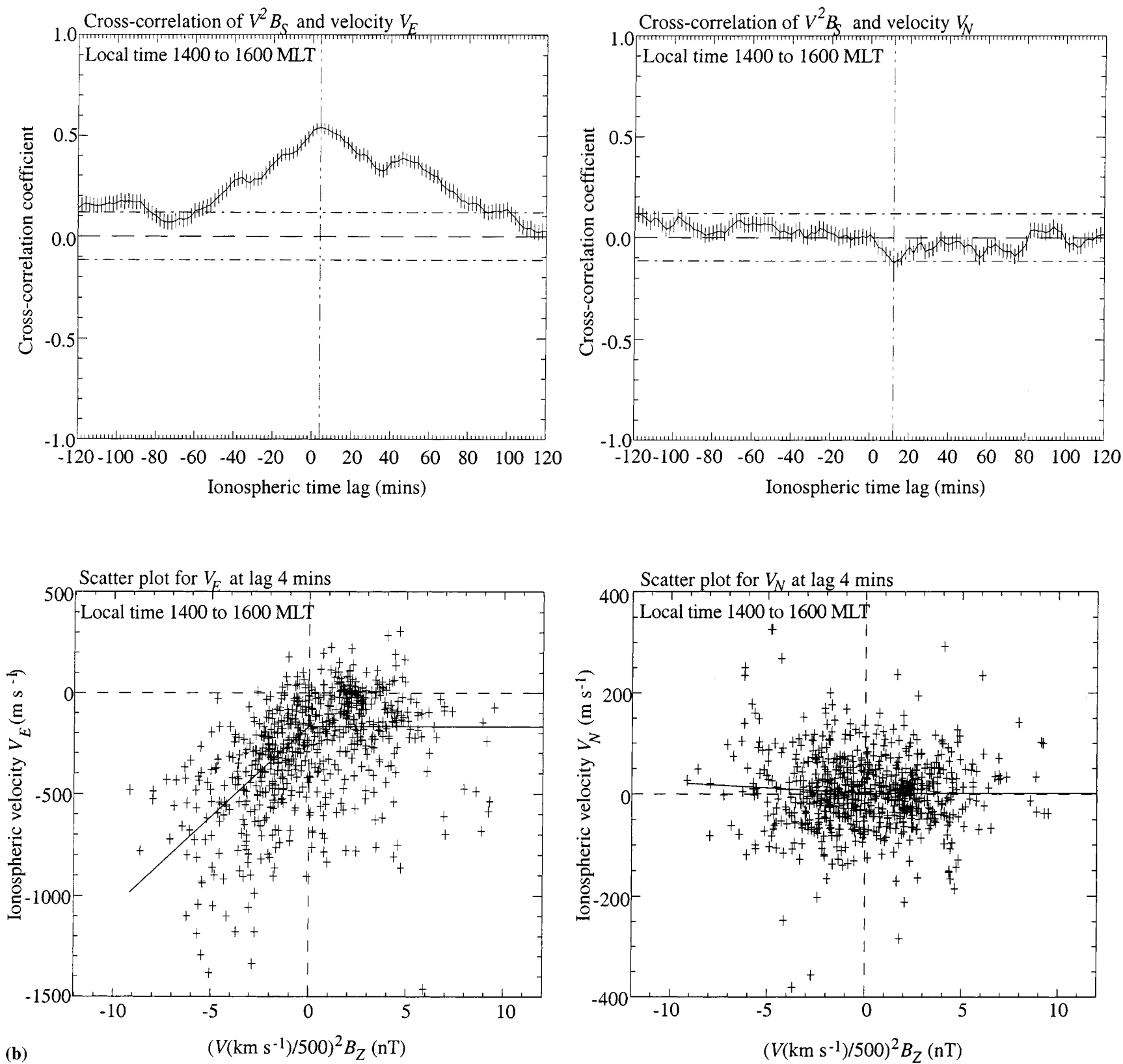

(b)

$\left(V\left(\mathrm{~km} \mathrm{~s}^{-1}\right) / 500\right)^{2} B_{Z}(\mathrm{nT})$

Fig. 4. b As for a except for the 2-h interval centred on 1500 MLT

is quantified from the error bar on the peak value by examining how many adjacent cross-correlation values lie within its limits. In this case four values lie within these limits at longer lags, and none at shorter lags. We thus take the "error bar" on the lag of the peak to lie between 2 and $10 \mathrm{~min}$. Secondary and even tertiary peaks are also evident in the cross-correlogram, on both sides of the main peak. Analysis indicates, however, that these are due to auto-correlation in the input interplanetary data at the corresponding periods, and are not due to secondary or tertiary response times in the ionospheric flow. The positive value of the cross-correlation coefficient at the peak implies the existence of westward ionospheric flow (i.e. negative $v_{E}$ ) when IMF $B_{z}$ is negative. This is confirmed by the scatter-plot shown beneath the cross-correlogram plotted for a lag of

2 min, which exhibits weak averaged westward flows for IMF $B_{z}$ positive (with no particular trend evident in the data) and increasing westward flows for IMF $B_{z}$ negative. Since the scatter-plot shows that the enhanced flows present for IMF $B_{z}$ negative are essentially consistently westward, becoming generally stronger as IMF $B_{z}$ becomes increasingly negative, it is appropriate according to the above discussion to interpret the lag time of the peak correlation as the flow excitation and decay delay time. This conclusion is also consistent with the small lag value ( $2 \mathrm{~min}$ ) obtained. That the east-west flow in the noon sector is consistently westward at EISCAT latitudes agrees with the results of Weimer (1995) shown in Fig. 1, where we see that the dusk cell generally extends across noon to give westward flow at noon at lower latitudes. A similar positive correlation 

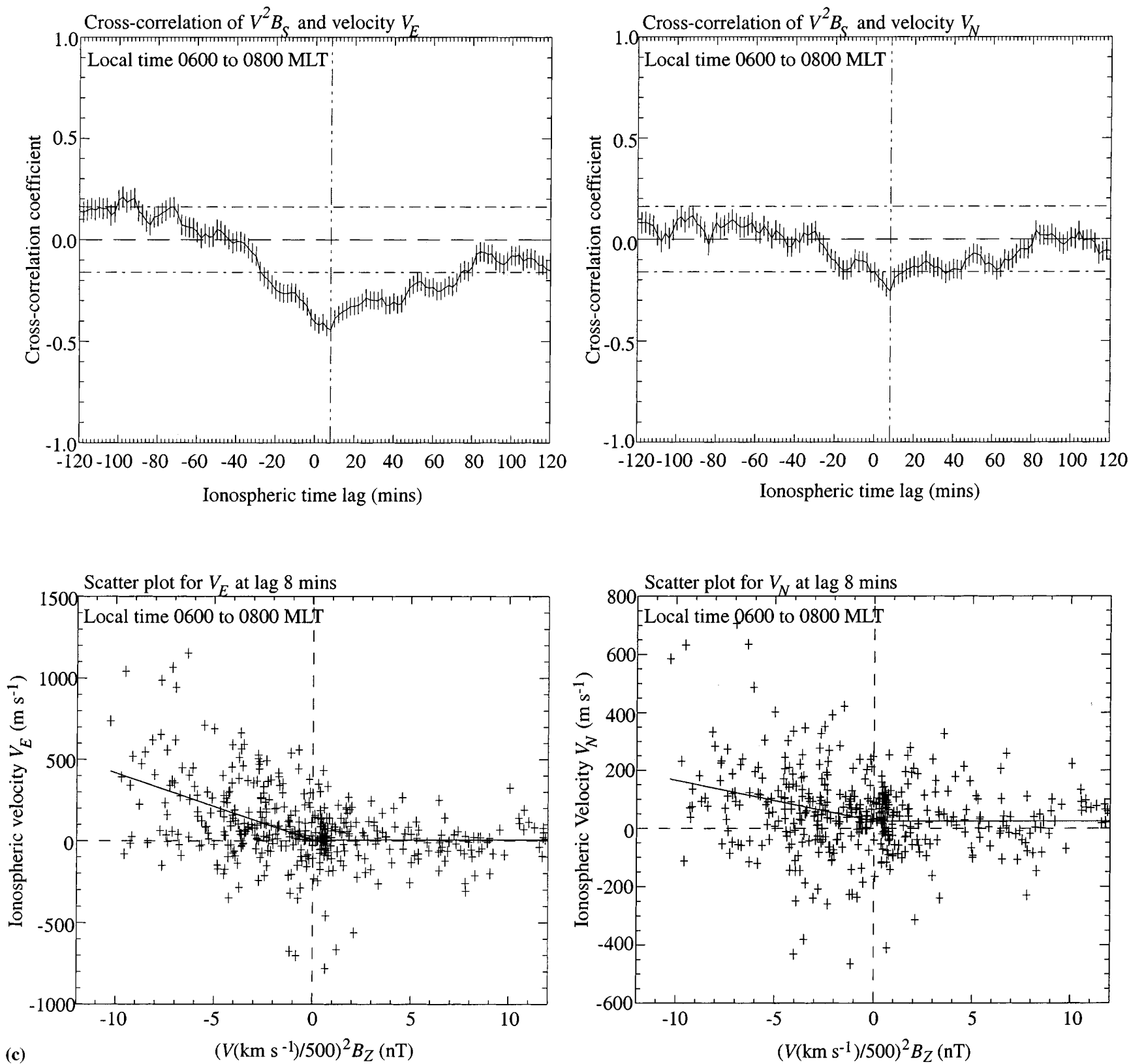

Fig. 4. c As for a except for the 2-h interval centred on 0700 MLT

and mainly westward flow is also found here in the MLT interval centred on 1100 MLT (not shown), reversing to a negative correlation associated with the eastward flow of the dawn cell at 0800 and earlier MLT (see later). No statistically significant correlation with $v_{E}$ is found for the intervals centred on 0900 and 1000 MLT.

The right side of Fig. 4a shows results for $v_{N}$ in the noon sector. The correlogram shows a clear negative minimum peaking at zero lag (with an "error bar" between -2 and $+2 \mathrm{~min}$ ), with a peak cross-correlation coefficient of -0.42 . The negative value implies increasing northward flows (positive $v_{N}$ ) for negative IMF $B_{z}$, as confirmed by the scatter plot underneath. Similar negative $v_{N}$ correlations with relatively short response delays, which we may interpret as the flow excitation and decay delay time, are found in the dayside hours over the interval 0700-1300 MLT, thus spanning the interval $\sim 3 \mathrm{~h}$ on either side of the east-west flow reversal at 0900-1000 MLT.

On moving away from noon into the afternoon sector, clear correlations remain with the westward component of the dusk cell flow, while the response of the northward component tends to disappear. Results for the 2-h interval centred on 1500 MLT are shown in Fig. 4b (containing 708 flow measurements), representative of the interval 1300-1700 MLT. The cross-correlogram for $v_{E}$ shows a positive maximum (westward flow for negative IMF $B_{z}$ ) at a lag of $4 \mathrm{~min}$ (with "error bars" between 0 and $10 \mathrm{~min}$ ), with a peak value of 0.54 . Again, two nearly symmetrical secondary peaks are also present, due to autocorrelation of the interplanetary input. The corresponding 

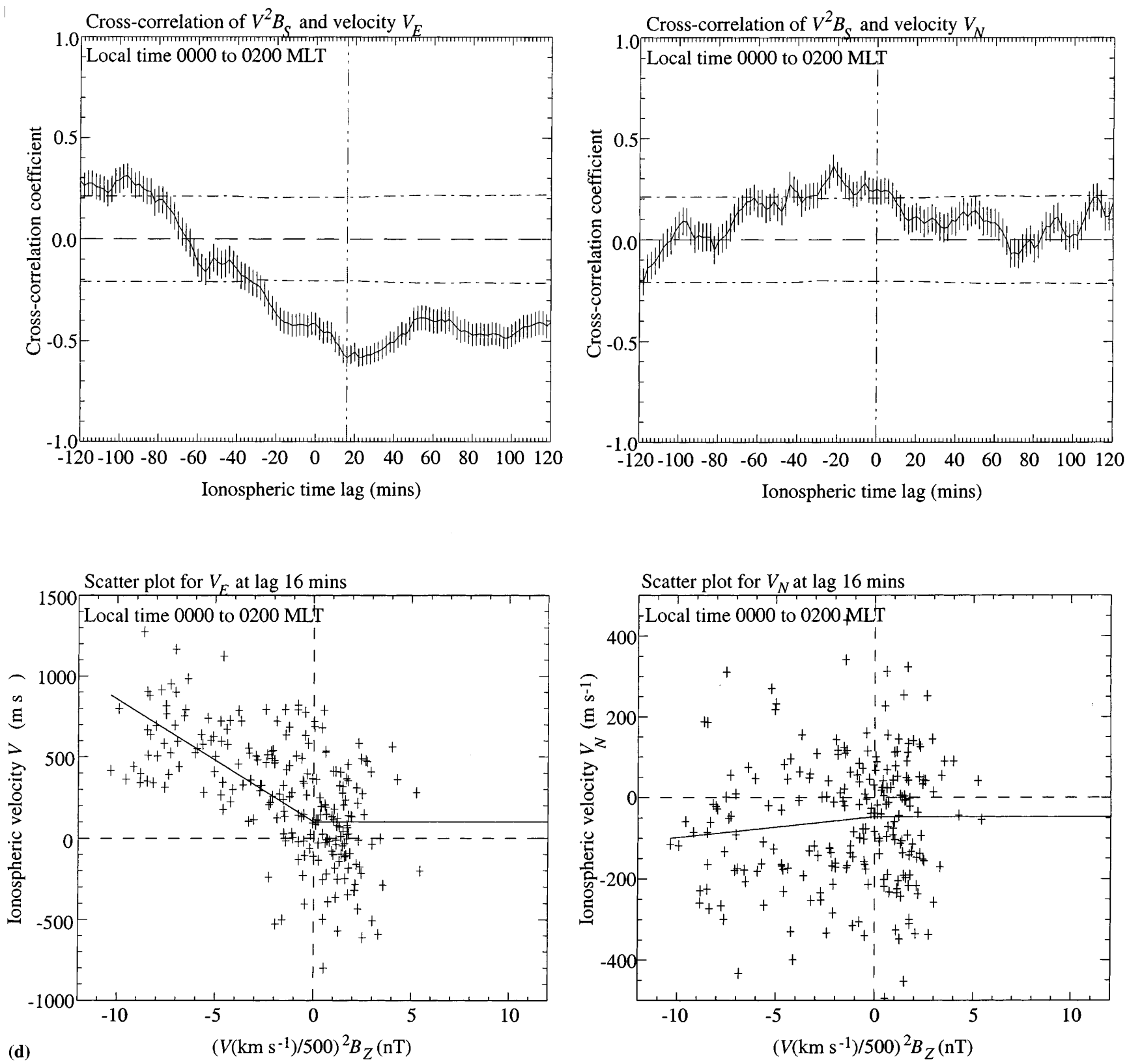

Fig. 4. d As for a except for the 2-h interval centred on 0100 MLT

scatter plot shows a flow that is now almost exclusively westward, which increases rapidly as IMF $B_{z}$ becomes more negative. Again, therefore, the lag time of the peak correlation can be interpreted as the excitation and decay delay time of the flow. The correlogram for $v_{N}$ on the right of Fig. 4b, however, shows no clear peak and no values above the $99.9 \%$ significance level. In this case the scatter plot is shown for the $4 \mathrm{~min}$ lag determined from $v_{E}$, and indicates an essentially zero averaged latitudinal flow in this local time sector, independent of IMF $B_{z}$. The averaged flow is thus almost purely westward in this local time sector.

Figure $4 \mathrm{c}$ shows related results for the dayside dawn cell, for the 2-h interval centred on 0700 MLT (containing 423 flow measurements). These are repre-

sentative of the results obtained in the interval 0500 0900 MLT, not previously covered in the study by Etemadi et al. (1988). The correlogram for $v_{E}$ now shows a negative minimum at a lag of 8 min (with an "error bar" between 0 and $8 \mathrm{~min}$ ), with a peak crosscorrelation coefficient of -0.44 . The scatter plot confirms that this correlation is related to an increasing eastward flow for IMF $B_{z}$ negative, with essentially zero averaged flow when IMF $B_{z}$ is positive. Again, the monotonic dependence of the flow component on IMF $B_{z}$ indicates that the lag of the peak correlation should be interpreted as the flow excitation and decay delay time. Similar results are obtained for $v_{N}$, with a negative peak cross-correlation value of -0.26 occurring at a lag of $8 \mathrm{~min}$ (with an "error bar" between 6 and $8 \mathrm{~min}$ ). This correlation is associated with modestly increasing 

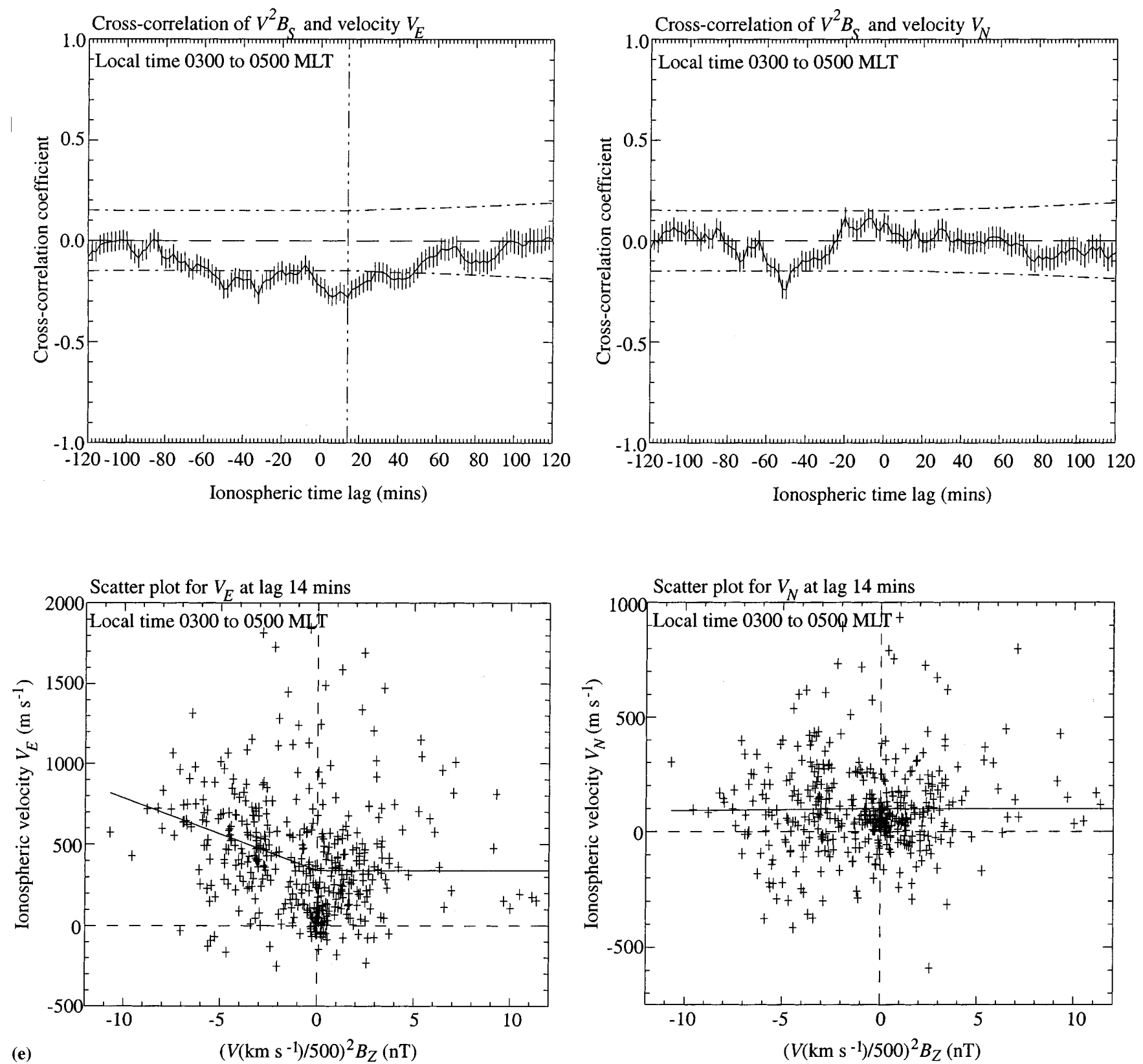

Fig. 4. e As for a except for the 2-h interval centred on 0400 MLT

northward flows for IMF $B_{z}$ negative, with small averaged flows for $B_{z}$ positive.

The dayside results surveyed above are indicative of a major control on the flow exerted by the north-south component of the IMF, with response delays at Tromsø of just a few minutes relative to the arrival of first effects at the ionosphere. Delay times are typically $2-4 \mathrm{~min}$ in the noon-afternoon sector of the dusk cell, with an indication of an increase to $\sim 8$ min near dawn. Results for the nightside are not so clear-cut. Typically, the cross-correlation coefficients are lower and the extrema less well defined, presumably because of the increasingly important influence on the flow of tail processes compared with magnetopause processes. Nevertheless, the results are indicative of increased response delays of $\sim 5-15 \mathrm{~min}$. Two examples from the post-midnight dawn cell are shown in Fig. $4 d$, e. Figure $4 d$ shows results for the 2-h interval centred on 0100 MLT (containing 225 flow measurements). Here the crosscorrelogram for $v_{E}$ has a pronounced but broad minimum, with a peak of -0.58 at a lag of 16 min (with an "error bar" between 14 and $34 \mathrm{~min}$ ). The scatter plot shows that this corresponds to eastward flows which increase strongly with increasingly negative IMF $B_{z}$ from small average values for IMF $B_{z}$ positive. No evidence for flow reversal effects are observed in these data, so that the lag time of the peak correlation should be interpreted as the excitation and decay delay time. The cross-correlogram for $v_{N}$ shows no pronounced peak for positive lags at values above the $99.9 \%$ level. 

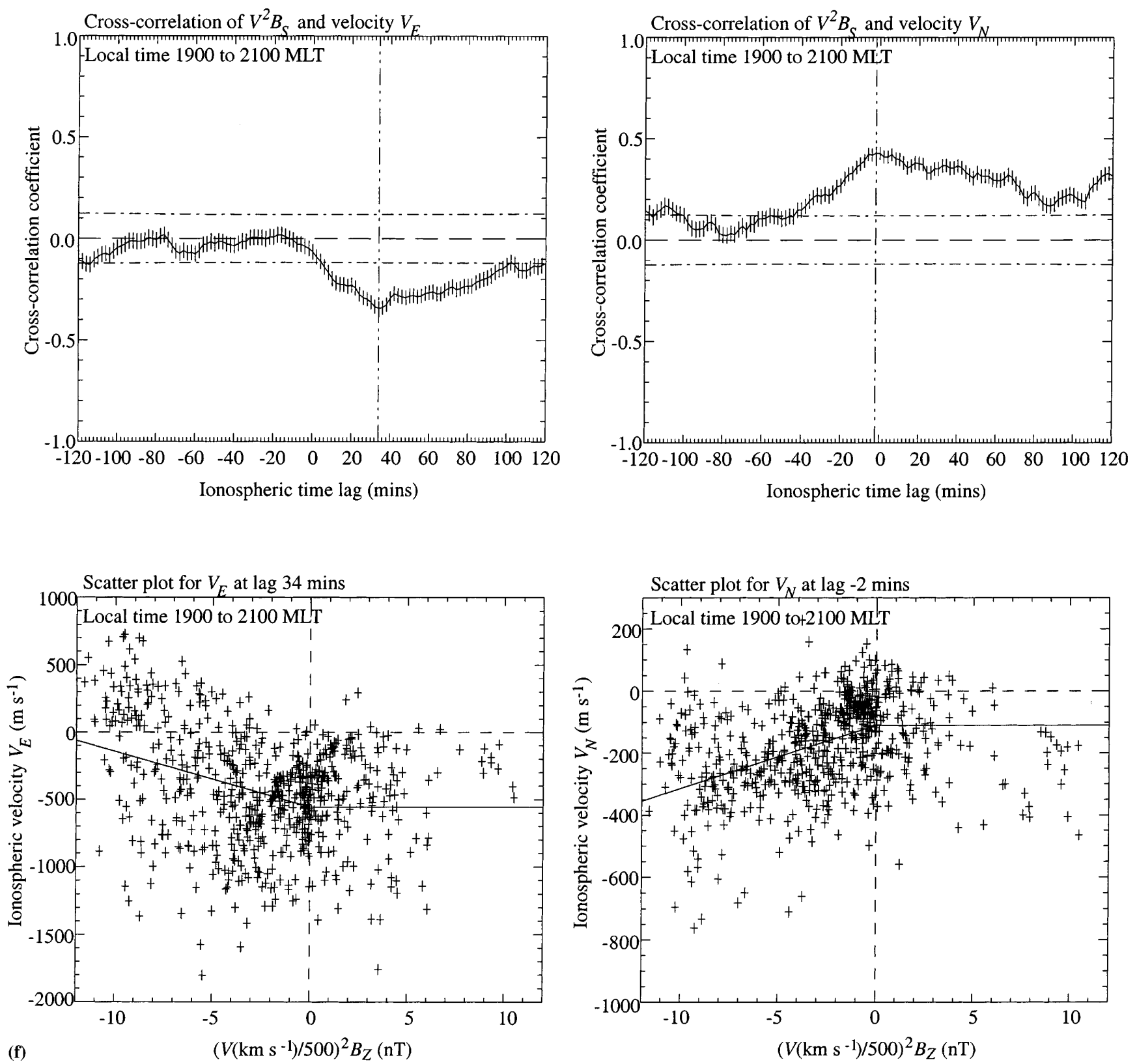

(f)

$\left(V\left(\mathrm{~km} \mathrm{~s}^{-1}\right) / 500\right)^{2} B_{Z}(\mathrm{nT})$

Fig. 4. f As for a except for the 2-h interval centred on 2000 MLT

The scatter-plot, shown at a lag of 16 min as determined from $v_{E}$, indicates weak averaged equatorward flows that barely respond to the IMF. Figure 4e similarly shows results for the 2-h interval centred on 0400 MLT (containing 425 flow measurements). Here the correlogram for $v_{E}$ has a weak minimum of -0.28 , but above the $99.9 \%$ confidence level, at a lag of 14 min (with a "error bar" between 2 and $16 \mathrm{~min}$ ). The scatter plot again shows consistently increasing eastward flows with increasing negative IMF $B_{z}$, though scattered large values are also present for IMF $B_{z}$ positive, perhaps due to tail (substorm) effects. As before, the cross-correlogram for $v_{N}$ shows no pronounced peak above the $99.9 \%$ confidence level. The scatter-plot at a lag of 14 min shows weak averaged northward flows, which are independent of the IMF north-south polarity.

The final MLT interval which we need to discuss is the sector from dusk to pre-midnight, i.e. the MLT hours centred on 1900-2300 MLT corresponding to the nightside region of the dusk cell. Here the results show rather different features, as exemplified by the data for the 2-h interval centred on 2000 MLT displayed in Fig. 4f (containing 682 flow measurements). Here the cross-correlogram for $v_{E}$ shows a broad but well-defined peak at a value of -0.38 with a delay of $34 \mathrm{~min}$ (with an "error bar" between 30 and $38 \mathrm{~min}$ ), a much longer delay than found in other local time sectors, as we have seen. Furthermore, the scatter-plot corresponding to this delay provides clear evidence of non-monotonic behaviour of the east-west flow with IMF $B_{z}$, the flow being westward for IMF $B_{z}$ positive, increasing in strength somewhat for small negative $B_{z}$, and then 
reversing in sense to become eastward for large negative $B_{z}$. Very similar behaviour is found in the 2-h interval centred on 1900 MLT, where the lag of the peak correlation again occurs with a delay of $34 \mathrm{~min}$ (within an "error bar" between 28 and $44 \mathrm{~min}$ ). From the previous discussion we may infer that this behaviour relates to the passage of the Harang east-west flow reversal across the observing site as the flow system expands with increasing negative IMF $B_{z}$. We then further infer that the lag of the peak correlation relates more to the time scale required for the sense of the flow to change relative to the IMF $B_{z}$ positive "baseline", and not to the excitation and decay delay time as in other local time sectors. The results of the "event" analysis presented in the next section support this interpretation by showing that the initial flow response in this MLT sector to individual sharp switches in the sense of IMF $B_{z}$ occur on significantly shorter time scales than those derived here from the cross-correlation analysis, and that they involve an enhancement in the westward flow when IMF $B_{z}$ turns from positive to negative ( 5 cases in the data set), and a reduction in the eastward flow when IMF $B_{z}$ turns from southward to northward ( 1 case in the data set). The cross-correlation results for $v_{E}$ in the MLT intervals centred on 21 and 22 MLT (not shown) are similar to those in Fig. 4f in that they show that a generally weak but westward flow which is present for IMF $B_{z}$ positive gives way to an increasing eastward flow when IMF $B_{z}$ is negative. However, the behaviour is now observed to be nearly monotonic relative to the positive IMF $B_{z}$ "baseline", with eastward flows appearing for relatively small negative values of IMF $B_{z}$, and with little evidence of significant initial enhancements of westward flow. Correspondingly, the lag of the peak correlation decreases towards values which are similar to those obtained at other local times, i.e. 14 min at 2100 MLT (within an "error bar" between 10 and $38 \mathrm{~min}$ ), and $10 \mathrm{~min}$ at 2200 MLT (within an "error bar" between 6 and $24 \mathrm{~min}$ ). It seems reasonable to infer that these values correspond to a measure of the excitation and decay delay time of the eastward flow in the pre-midnight sector.

We now turn to the results for $v_{N}$ in the pre-midnight Harang region, where, since the considerations which apply to the reversing $v_{E}$ component no longer hold, we may again suppose a priori that the lag time of the peak correlation will correspond to the excitation and decay delay time. Correspondingly, the correlogram for $v_{N}$ for the interval centred on 2000 MLT shown in Fig. 4f does not show a peak at the same lag as for $v_{E}$, but rather exhibits a very broad region of positive correlation with a peak of 0.43 at an (unphysical) lag of $-2 \mathrm{~min}$ (within an "error bar" between -4 and $+10 \mathrm{~min}$ ). The $v_{N}$ scatter-plot shows a well-defined and monotonic increase in equatorward flow with increasing negative IMF $B_{z}$. Similar $v_{N}$ behaviour is found in the interval centred on 1900 MLT, but this effect dies away with increasing local time, and no significant correlations are found for the interval centred on 2100 MLT and at later local times.
We will now summarise the outcome of the crosscorrelation analysis, beginning in Fig. 5a by showing results for the eastward component of ionospheric flow. The top panel firstly shows the number of flow measurements in each 2-h interval of MLT, varying between a minimum of about 200 in the post-midnight sector, to a maximum of about 700 in the afternoon and evening sector, as previously indicated. Beneath this we show the response delay of the eastward flow component versus MLT, corresponding to the lag of the peak cross-correlation coefficient, with error bars determined as described. No value is shown if there was no peak present above the $99.9 \%$ confidence level in the crosscorrelogram. Below this we show the peak value of the cross-correlation coefficient itself. The lower two panels then show the slope and intercept of the least-squares lines fitted to the scatter plots at the lag of the peak correlation (as shown in the lower graphs in Fig. 4). The "intercept" velocity provides a measure of the average flow which is present for northward IMF, while the "gradient" indicates how this baseline value changes in response to various levels of negative IMF $B_{z}$.

In line with the prior discussion, the results for $v_{E}$ can be divided into three main regimes. The first is the dayside dusk cell westward flow regime indicated by the square symbols, observed between 1100 and 1700 MLT. Here the flow response delay is typically $2-4 \mathrm{~min}$, and shows no discernible MLT variation within the resolution of this study. The cross-correlation coefficients are typically quite high, between $\sim 0.4$ and $\sim 0.7$, and tend to increase with local time. The "gradient" values also increase with MLT, corresponding to radar locations which are increasingly centrally located within the dusk flow cell, as do the "intercept" velocities, though the latter remain quite small, less than $\sim 200 \mathrm{~m} \mathrm{~s}^{-1}$. The second regime corresponds to the dawn cell eastward flow region indicated by the circular symbols, observed between 0000 and 0800 MLT. Here the flow response delay times are short, $\sim 5 \mathrm{~min}$, and similar to the dusk cell values on the dayside (certainly within the error bars), but tend to increase and become more scattered, 5-15 min, on the nightside. The peak cross-correlation coefficients are lower than for the dusk cell, typically $\sim 0.3-0.4$, while in common with the dusk cell, the "gradient" and "intercept" values tend to increase on moving from the dayside to the nightside, before declining somewhat near midnight. The third regime corresponds to the nightside (Harang) region of the dusk cell, observed between 1900 and 2200 MLT, marked by crosses. As noted already, this regime is characterised by the presence of westward flow for northward IMF, reversing to eastward flow for a sufficiently southward IMF, such that the "gradient" and "intercept" are of the same sign, unlike the opposite signs of the other two regions. Here the response delays are very long ( $\sim 30-40 \mathrm{~min})$ at 1900 and 2000 MLT, but decline to $\sim 10-15 \mathrm{~min}$ at 2100 and $2200 \mathrm{MLT}$ as outlined. As previously indicated, the lag times of the peak correlation in the interval 1900-2000 MLT probably more reflect the time scale for flow reversal from west to east due to expansion and evolution of the flow 


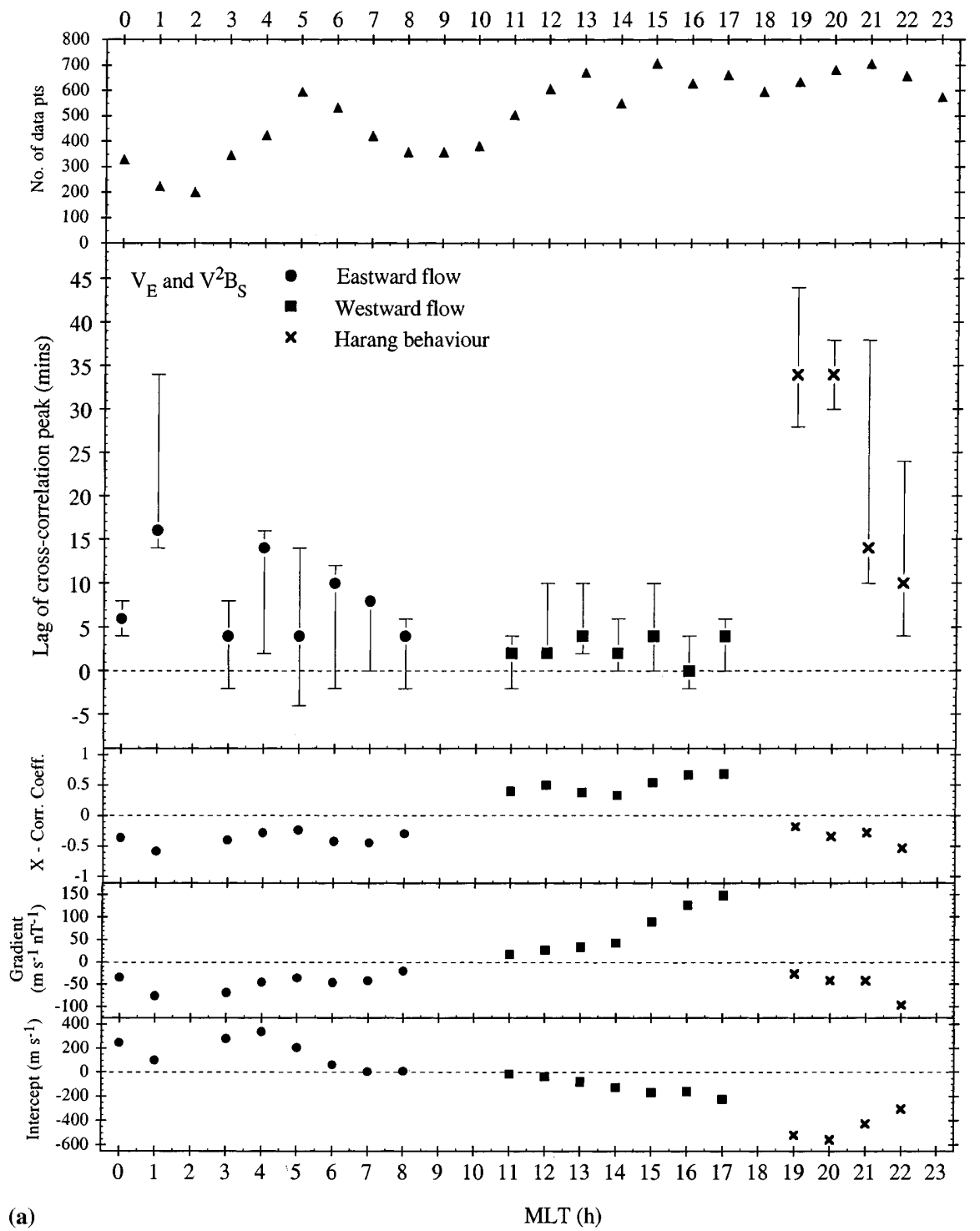

(a)

squares indicate results corresponding to the dayside dusk flow cell where the correlation coefficient and "gradient" are positive, while the "intercept" is negative (increasing westward flows for southward IMF). The circles indicate results corresponding to the dawn flow cell where the correlation coefficient and "gradient" are negative, while the "intercept" is positive (increasing eastward flows for southward IMF). The crosses indicate the Harang region of the nightside dusk flow cell where the correlation coefficient, "gradient", and "intercept" are all negative (flow reverses from west to east as the IMF reverses from north to south).

cells under the influence of southward IMF, rather than to the IMF-modulated flow excitation and decay time scale of main interest here. These two values will consequently be excluded from further analysis of the latter delay time.

Results for $v_{N}$ shown in Fig. $5 \mathrm{~b}$ are more restricted, due to the fact that within the central parts of the flow cells the north-south flow tends to be weak and unresponsive to the IMF. Two regimes are evident, corresponding to the region of negative correlations indicated by the square symbols (northward flow for southward IMF), which span the dayside east-west flow reversal centred on 0900-1000 MLT, and a region of

positive correlations indicated by the circles (equatorward flow for southward IMF), which are observed in the dusk and early evening sector. The noon-sector $v_{N}$ data indicate similar short response delays as found for $v_{E}$, and bridge the "gap" in the latter results in the interval where the east-west flow reverses. Typical crosscorrelation coefficients are fairly low, $\sim-0.3$. Values of the "gradient" tend to be higher in the morning than near noon, and the "intercept" remains small throughout. Short response delays are also indicated in the region of positive correlation (equatorward flow) in the dusk and post-dusk sector, though the correlation distributions tend to be very broad (Fig. 4f) and the 


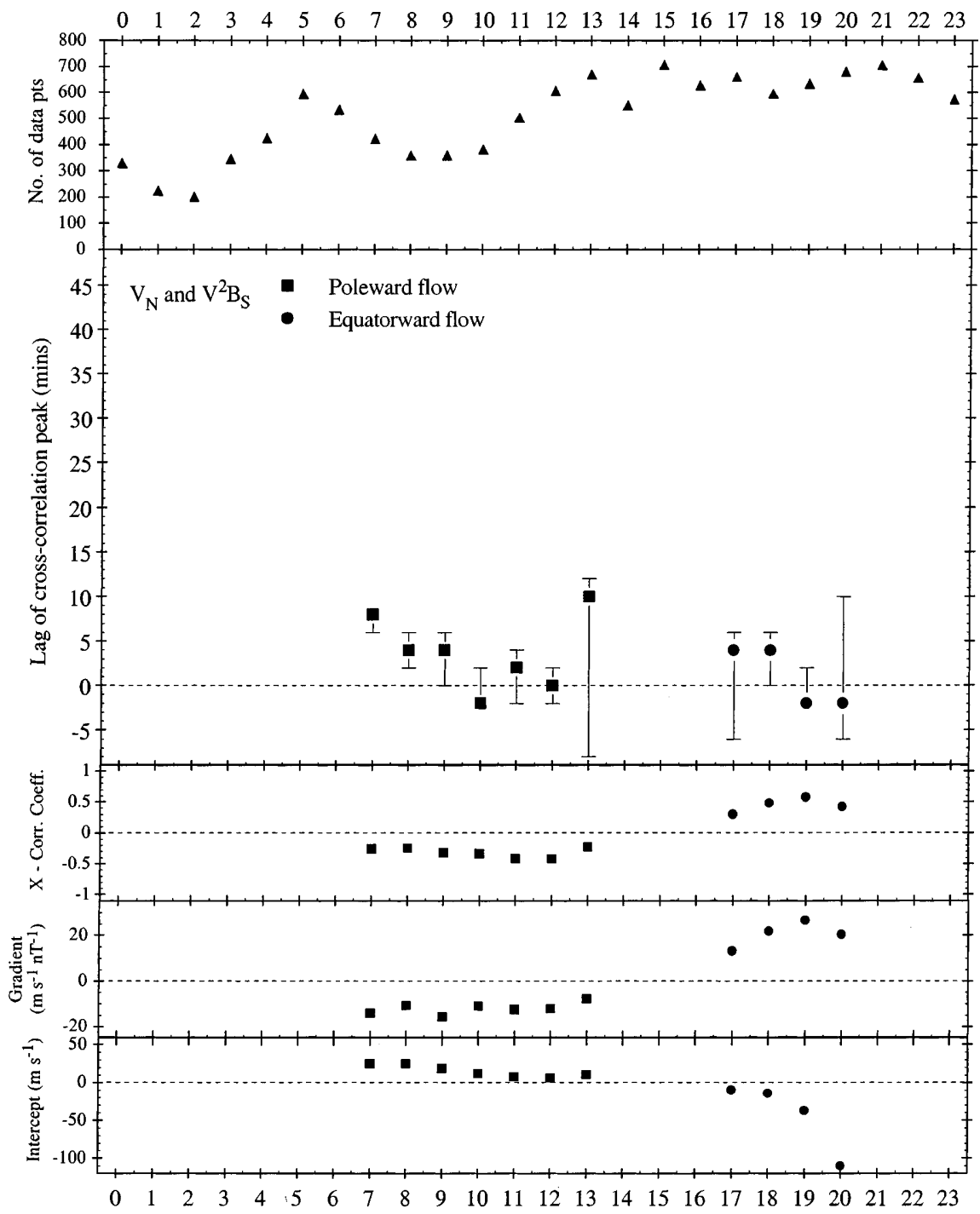

(b)

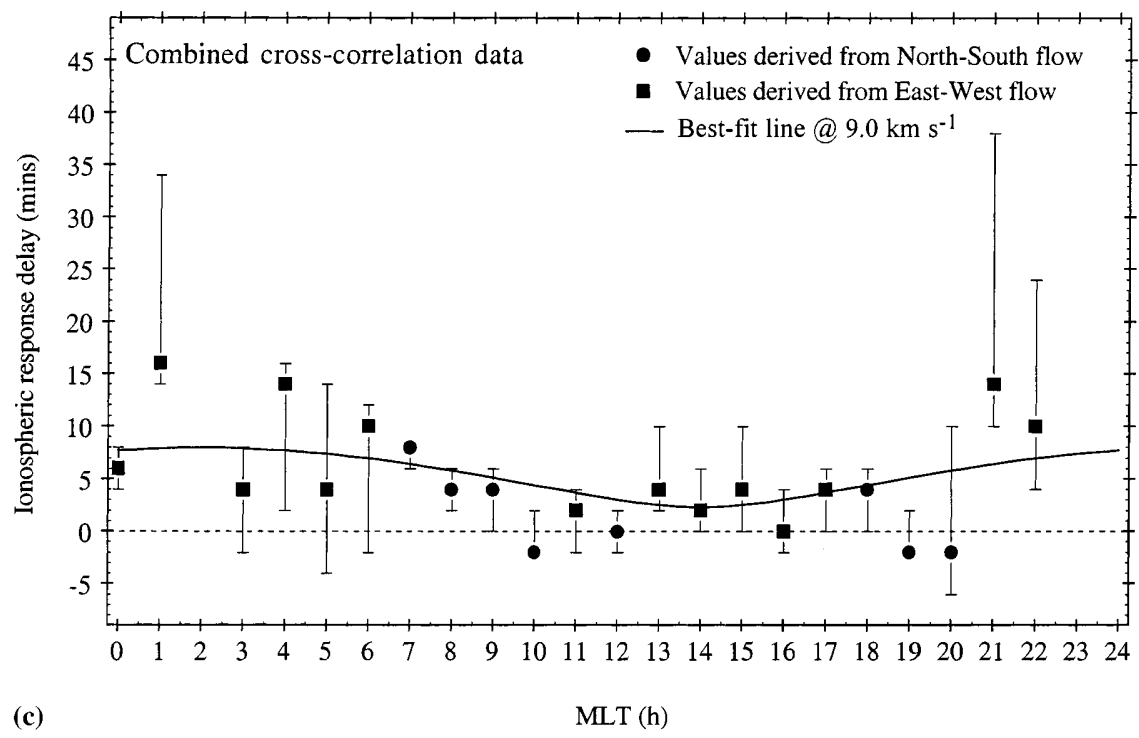

Fig. 5. b As for a, except for the $v_{N}$ component of ionospheric flow. The squares indicate the region of negative cross-correlation values (northward flow for southward IMF), while the circles indicate the region of positive values (equatorward flow for southward IMF)

Fig. 5. c Response delay values for the combined north-south and east-west cross-correlation data set, where the squares indicate values derived from the east-west flow, and circles values derived from the north-south flow. Where both components provide a value, the lower of the two has been used, and where both give the same value, the data point with the smaller error bars is shown. The solid line shows a simple theoretical curve which has been leastsquares fit to these values, in which information propagates radially from the open-closed field line boundary at $77^{\circ}$ magnetic latitude. The bestfit line corresponds to a propagation epicentre located at 1400 MLT, and a phase speed of $9.0 \mathrm{~km} \mathrm{~s}^{-1}$ 
uncertainties large. Cross-correlation values peak at $\sim 0.6$ at 1900 MLT, and "gradient" and "intercept" values tend to increase with increasing local time from dusk towards midnight.

Considering all of the $v_{E}$ flow response results shown in Fig. 5a, it can be seen that the data are consistent with short, few-minute response delays on the dayside, and with increased delays on the nightside, though the scatter in the latter values is large. If we take all of the dayside values from 0700 to 1700 MLT and weight them according to the inverse of the error bar to take account of their varying degrees of certainty, we find an average delay time of $3.1 \pm 0.8 \mathrm{~min}$ (the unweighted average is $3.3 \pm 0.8 \mathrm{~min}$ ). (These averages are collected together and displayed in Table 1.) For the nightside values from 2100 to 0500 MLT (excluding the two values at 1900 and 2000 MLT for reasons discussed) we find a significantly higher weighted average value of $8.0 \pm 2.4 \mathrm{~min}$ (the unweighted average is $9.7 \pm 1.9 \mathrm{~min}$ ). Here the errors are quoted as the standard error of the weighted mean (i.e. the standard deviation divided by the root of the number of samples in the case of equal weights). For the north-south component of the flow, the dayside data shown in Fig. 5b confirm the short response delays which are present in the noon sector. The weighted average response delay of the $v_{N}$ values in the interval 0700-1300 MLT is found to be $3.7 \pm 1.8 \mathrm{~min}$, entirely consistent with the dayside results for $v_{E}$. The $v_{N}$ results in the dusk sector also indicate short, few-minute delays. These data are comparable to the values obtained from $v_{E}$ in the pre-dusk sector, but are much shorter than the $\sim 30-40$ min values obtained from $v_{E}$ in the post-dusk sector, for reasons already discussed.

Although the discussion has concentrated on the cross-correlation results for the two flow components taken separately, it is also of interest to combine the results together. This significantly improves the MLT coverage, since, as we have seen, the flow responds principally in the east-west component in some sectors, and principally in the north-south component in others.
At local times where both components provide an estimate of the flow response delay, we have taken the smaller of the two values on the basis that the true response delay for the flow in a particular MLT sector corresponds to the minimum value obtained, irrespective of the component in which it was observed. The results are shown in Fig. 5c, where square symbols indicate values derived from the east-west flow component, while circles indicate values derived from the north-south flow component; where both components give the same value we show the point with the smallest error bar. Again the results suggest longer delays on the nightside compared with the dayside, with the weighted average of the dayside values being $2.2 \pm 0.9 \mathrm{~min}$ $(2.7 \pm 0.8 \mathrm{~min}$ unweighted), while that for the nightside being $5.5 \pm 2.5 \mathrm{~min}(7.1 \pm 2.3 \mathrm{~min}$ unweighted). However, the results taken together suggest that the minimum response delay actually occurs in the post-noon sector, near $1400 \mathrm{MLT}$, rather than at noon itself. Although the scatter in this data set is rather large, the reality of the offset from noon is suggested by the fact that a very similar effect was previously observed in the cross-correlation and event analyses performed by Etemadi et al. (1988) and Todd et al. (1988), respectively, using EISCAT flow data measured at higher latitudes $\left(71^{\circ}-73^{\circ}\right)$. It also occurs in the event analysis presented here in the following section. If we then average the data in Fig. 5c over the 12-h sectors centred on 1400 MLT and $0200 \mathrm{MLT}$, the weighted averages are found to be $1.3 \pm 0.8 \mathrm{~min}$ and $8.8 \pm 1.7 \mathrm{~min}$, respectively (see Table 1). (The unweighted values are $1.8 \pm 0.7 \mathrm{~min}$ and $9.6 \pm 1.5 \mathrm{~min}$, respectively.) The difference between "dayside" and "nightside" response delays is thus estimated as $7.5 \pm 1.9 \mathrm{~min}$.

The results shown in Fig. 5c suggest that at the latitude of EISCAT, at least, IMF-modulated changes in the flow are observed first in the immediate post-noon sector, and then propagate into the nightside, such that the response is delayed in the latter region relative to the former. In order to give an indication of the effective

Table 1. Averaged flow response delays in different local time sectors derived from the various data sets employed in this study. For the cross-correlation results the contributing data points have been weighted according to the inverse of their uncertainty estimate

\begin{tabular}{|c|c|c|c|}
\hline Data set & $\begin{array}{l}\text { Dayside response delay } \\
\text { (range of MLT) }\end{array}$ & $\begin{array}{l}\text { Nightside response delay } \\
\text { (range of MLT) }\end{array}$ & $\begin{array}{l}\text { Nightside-dayside } \\
\text { delay difference }\end{array}$ \\
\hline$v_{E}$ cross-correlation data & $\begin{array}{l}3.1 \pm 0.8 \mathrm{~min} \\
(07-17 \mathrm{MLT})\end{array}$ & $\begin{array}{l}8.0 \pm 2.4 \mathrm{~min} \\
(21-05 \mathrm{MLT})\end{array}$ & $4.9 \pm 2.6 \mathrm{~min}$ \\
\hline$v_{E}$ event analysis data & $\begin{array}{l}5.4 \pm 0.5 \mathrm{~min} \\
(06-18 \mathrm{MLT})\end{array}$ & $\begin{array}{l}11.6 \pm 1.4 \mathrm{~min} \\
(18-06 \mathrm{MLT})\end{array}$ & $6.2 \pm 1.5 \mathrm{~min}$ \\
\hline$v_{N}$ cross-correlation data & $\begin{array}{l}3.7 \pm 1.8 \mathrm{~min} \\
(07-13 \mathrm{MLT})\end{array}$ & - & - \\
\hline Combined cross-correlation data & $\begin{array}{l}2.2 \pm 0.9 \mathrm{~min} \\
(07-17 \mathrm{MLT})\end{array}$ & $\begin{array}{l}5.5 \pm 2.5 \mathrm{~min} \\
(19-05 \mathrm{MLT})\end{array}$ & $3.3 \pm 2.9 \mathrm{~min}$ \\
\hline Combined event analysis data & $\begin{array}{l}4.8 \pm 0.5 \mathrm{~min} \\
(06-18 \mathrm{MLT})\end{array}$ & $\begin{array}{l}8.7 \pm 1.1 \mathrm{~min} \\
(18-06 \mathrm{MLT})\end{array}$ & $3.9 \pm 1.2 \mathrm{~min}$ \\
\hline Combined cross-correlation data & $\begin{array}{l}1.3 \pm 0.8 \mathrm{~min} \\
(09-19 \mathrm{MLT})\end{array}$ & $\begin{array}{l}8.8 \pm 1.7 \mathrm{~min} \\
(21-07 \mathrm{MLT})\end{array}$ & $7.5 \pm 1.9 \mathrm{~min}$ \\
\hline
\end{tabular}


phase speed of that propagation we have employed a simple model in which, starting at zero delay time, the information propagates radially outwards from the dayside open-closed field line boundary, taken to lie typically at $\sim 77^{\circ}$ magnetic latitude (according to observations of the location of the equatorward border of the cusp reported by Newell et al., 1989). The propagation speed and the local time of the epicentre of information propagation have then been stepped (through half-hour intervals of local time) until the least squares best fit to the cross-correlation data in Fig. 5c has been found. This procedure yields an epicentre located at 1400 MLT (thus validating the division of the data chosen), and a phase speed of $9.0 \mathrm{~km} \mathrm{~s}^{-1}$. The best-fit line is superposed on the data in Fig. 5c. It is seen to follow the trends in the data tolerably well, though we would hesitate to call it a "good fit" due to the large error bars and the consequent scatter in the data.

\section{Event analysis}

In order to complement the results of the crosscorrelation analysis reported, we here present the results of an "event analysis" undertaken in the manner of Todd et al. (1988), using the same data set as earlier. We have looked for the occurrence of sharp polarity changes in the IMF $B_{z}$ component, and have then examined concurrent measurements of the ionospheric flow to find the time at which the first response was observed, relative to the arrival time of first possible IMF effects. The latter time was determined from the IMP-8 interplanetary data in essentially the same way as for the cross-correlation analysis (see Appendix), except that the orientation of the "phase front" of the IMF was determined and employed for each "event" individually, with the delay time calculated from solar wind plasma data averaged over the same 10-15 min intervals on either side of the "event" (the IMF discontinuity) as were used to average the field for the "phase front" (discontinuity normal) analysis. The time of the ionospheric flow response was then determined as the centretime of the 2-min radar data averaging interval that showed the first enduring change that could be ascribed to the effect of the IMF, outside of the range of previous fluctuations of the flow data. Overall, the responses to a total of 69 such interplanetary "events" were analysed in this study.

We first show a few individual examples in various local time sectors before describing the overall results. Two "southward turn" events observed when EISCAT was in the noon sector were previously discussed in Sect. 2 and displayed in Fig. 2, where response delays in the westward flow component of $3 \pm 1 \mathrm{~min}$ were determined, consistent with the results of the crosscorrelation analysis in this local time sector (Fig. 5a). Here (Fig. 6a) we therefore begin by showing data for the interval 1200-1400 UT on 16 March, 1994, corresponding to the mid-afternoon sector (1430-1630 MLT) of the dusk flow cell (see also the correlation results in Fig. 4b). The format is the same as for Fig. 2. Two periods of predominantly southward fields can be seen in the IMF, observed by IMP-8 in the intervals 12131229 UT and 1304-1329 UT, respectively, as indicated by the vertical dashed lines in the upper panel. (We neglect short-term variations of the field, on times scales less than $\sim 5 \mathrm{~min}$, to which no ionospheric response can be discerned.) The effect of the first southward turn at 1213 UT is estimated to have arrived at the ionosphere at 1225 UT (a delay of $12 \mathrm{~min}$ relative to IMP-8, indicated by the first vertical dashed line in the lower panels), and is clearly associated with the onset of a flow which is directed westward and (weakly) equatorward. The response delay is somewhat obscured in this case by a one-point data gap centred at $1223 \mathrm{UT}$, but when data is resumed 1225 UT the weak equatorward flow is clearly already in progress, while the westward flow is not clearly increasing consistently and at larger values than those occurring previously until 1229 UT. The response delay of $v_{N}$ is therefore taken to be essentially zero, while that of $v_{E}$ is about $4 \mathrm{~min}$. The effect of the subsequent northward turn of the IMF, observed by IMP-8 at 1229 UT (second dashed line in the upper panel), is estimated to have arrived at the ionosphere at 1240 UT (second dashed line in the lower panels). The cessation of the perturbation in $v_{N}$ is rather gradual, from which no timing information is deduced, while the first time at which the westward flow is observed to decline (outside the range of general fluctuations in the data) occurs at $1245 \mathrm{UT}$, with a delay of $5 \mathrm{~min}$. The westward flow then died away gradually from $\sim 500$ to $\sim 100 \mathrm{~m} \mathrm{~s}^{-1}$ over an interval of $\sim 20 \mathrm{~min}$, as previously observed in this MLT sector by Todd et al. (1988). The IMF then turned south again at 1304 UT, with effects reaching the ionosphere at 1314 UT (third dashed lines). The first measurements exhibiting a clear enhancement in the flow are centred at 1319 UT in $v_{N}$, with a delay of $5 \mathrm{~min}$, and at $1317 \mathrm{UT}$ in $v_{E}$, with a delay of $3 \mathrm{~min}$. Finally, an enduring northward turn took place at 1329 UT, reaching the ionosphere at 1341 UT (fourth dashed lines). Both flow components show a first clear response at $1343 \mathrm{UT}$, with a delay of $2 \mathrm{~min}$, declining to small values over a $\sim 10 \mathrm{~min}$ interval. Overall, therefore, we infer small response delays in both flow components of typically $\sim 5 \mathrm{~min}$ or less. These individual observations are therefore entirely compatible with the results of the cross-correlation analysis in this MLT sector discussed (Fig. 4b), which show peak correlations with a delay of 4 min for the east-west flow component, though there are no significant responses in the north-south flow in the data set as a whole.

In Fig. 6b we show corresponding data for the dayside dawn flow cell, for the interval 0420-0620 UT (0650-0850 MLT) on 19 June, 1996. Here sharp southward turns of the IMF were observed by IMP-8 at 0454 , 0517, and 0542 UT (the second, third, and fifth dashed lines in the upper panel), which lead to excitation of relatively weak eastward and poleward flows (essentially simultaneously) at 0509, 0539, and 0601 UT (related dotted lines in the lower panels), with delays relative to first possible effects (related dashed lines in the lower panels) of 5, 12, and $8 \mathrm{~min}$, respectively. Sharp north- 


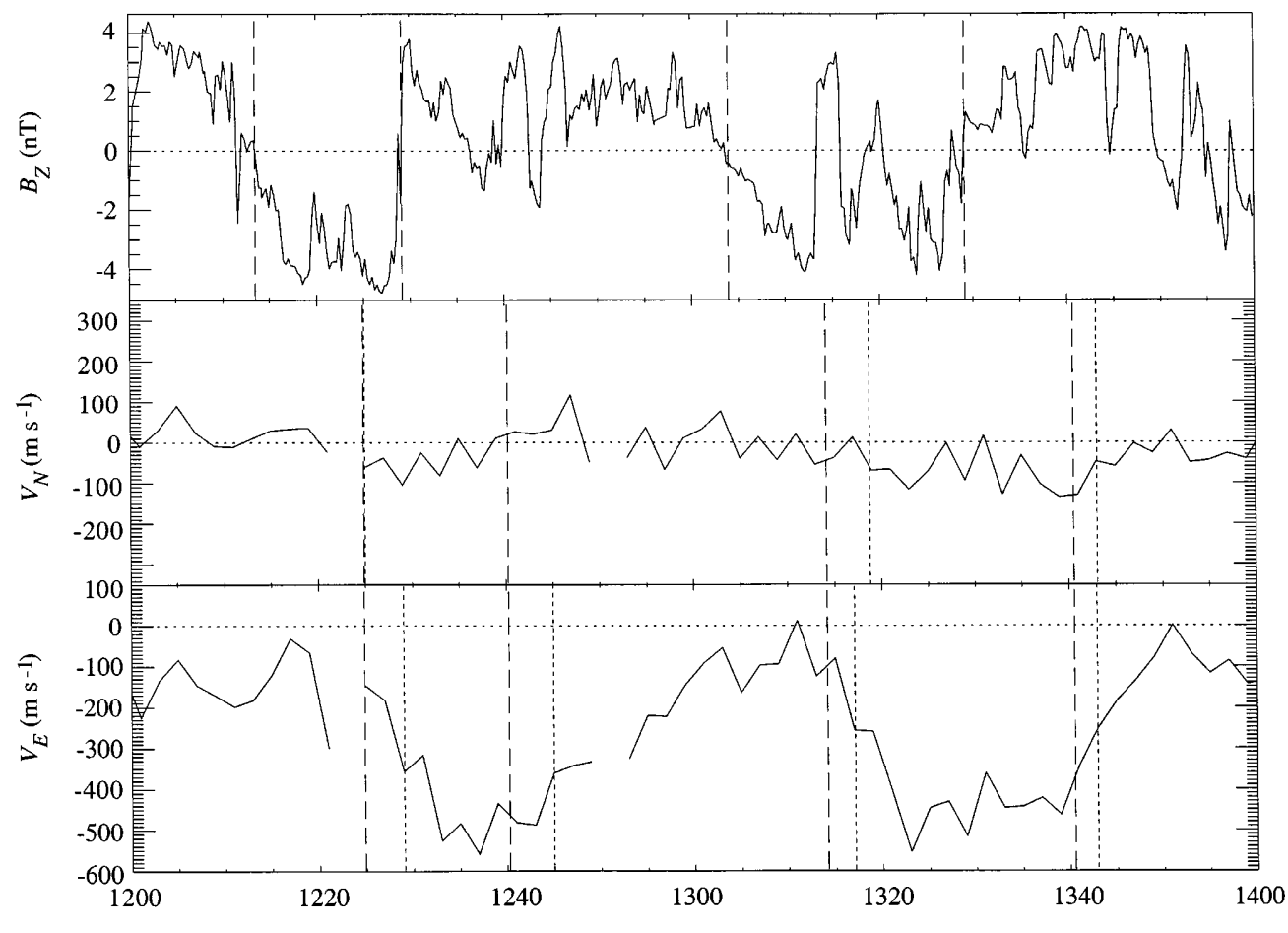

(a)

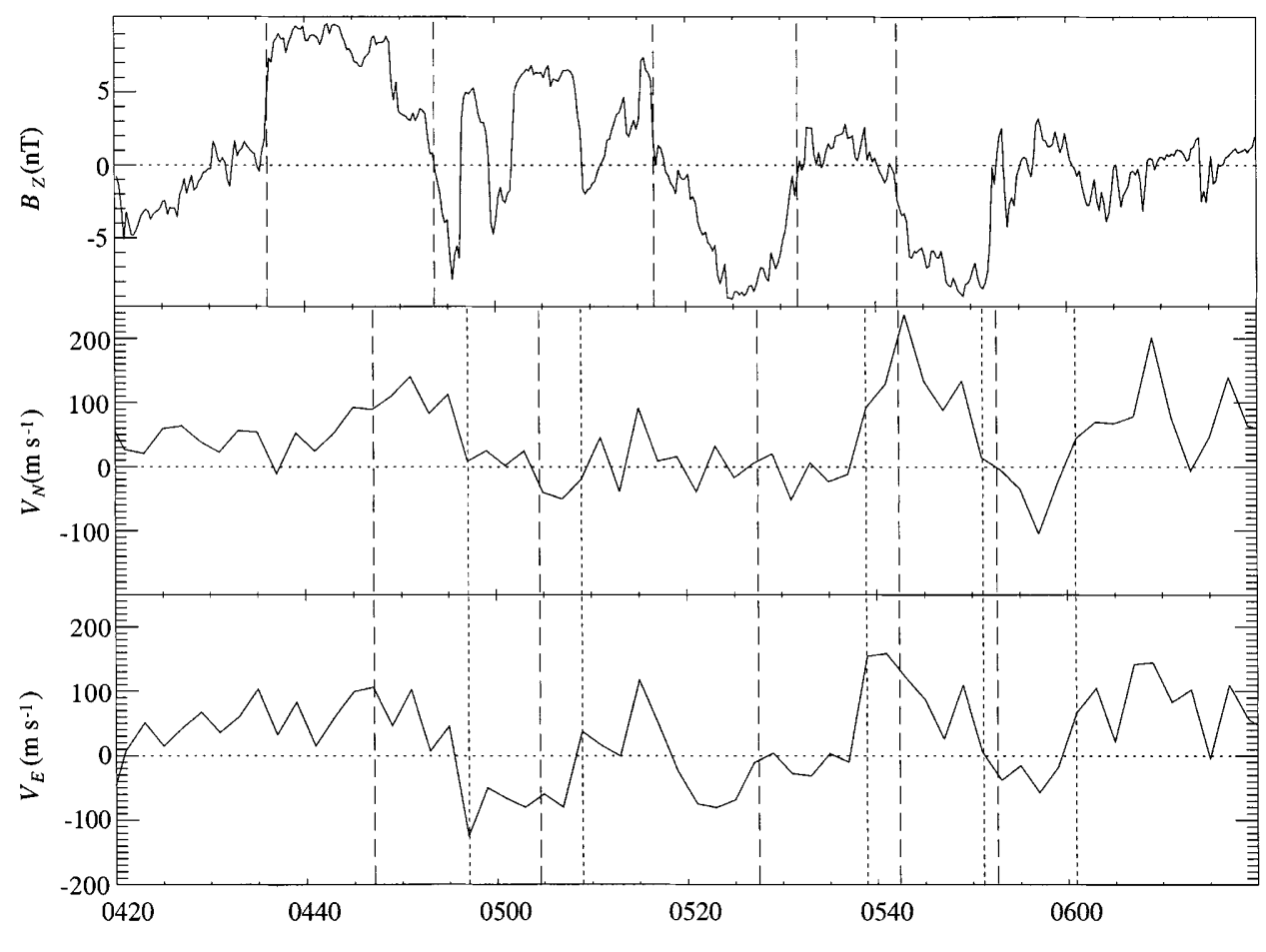

Fig. 6. a As for Fig. 2, except for the interval 1200-1400 UT (1430-1630 MLT) on 16 March, 1994
Fig. 6. b As for Fig. 2, except for the interval 0420-0620 UT (06500850 MLT) on 19 June, 1996

(b)

ward turns were also observed at 0436 and 0531 UT (the first and fourth set of vertical lines), leading to decay of those flows starting at 0457 and $0551 \mathrm{UT}$, with delays relative to first effects of 10 and $9 \mathrm{~min}$, respectively. Response delays are thus typically $\sim 10 \mathrm{~min}$ in this interval, compatible with the cross-correlation results for the post-dawn flow shown previously in Figs. 4c and 5.
Figure 6c shows results for an interval spanning midnight, 2045-2245 UT (2315-0115 MLT) on 15 March, 1994. Several potentially corresponding IMF and flow features appear during this interval, including the flow pulse centred on $\sim 2135$ UT which may be related to a brief interval of southward field beginning at $\sim 2120$ UT. However, we restrict attention here to the 

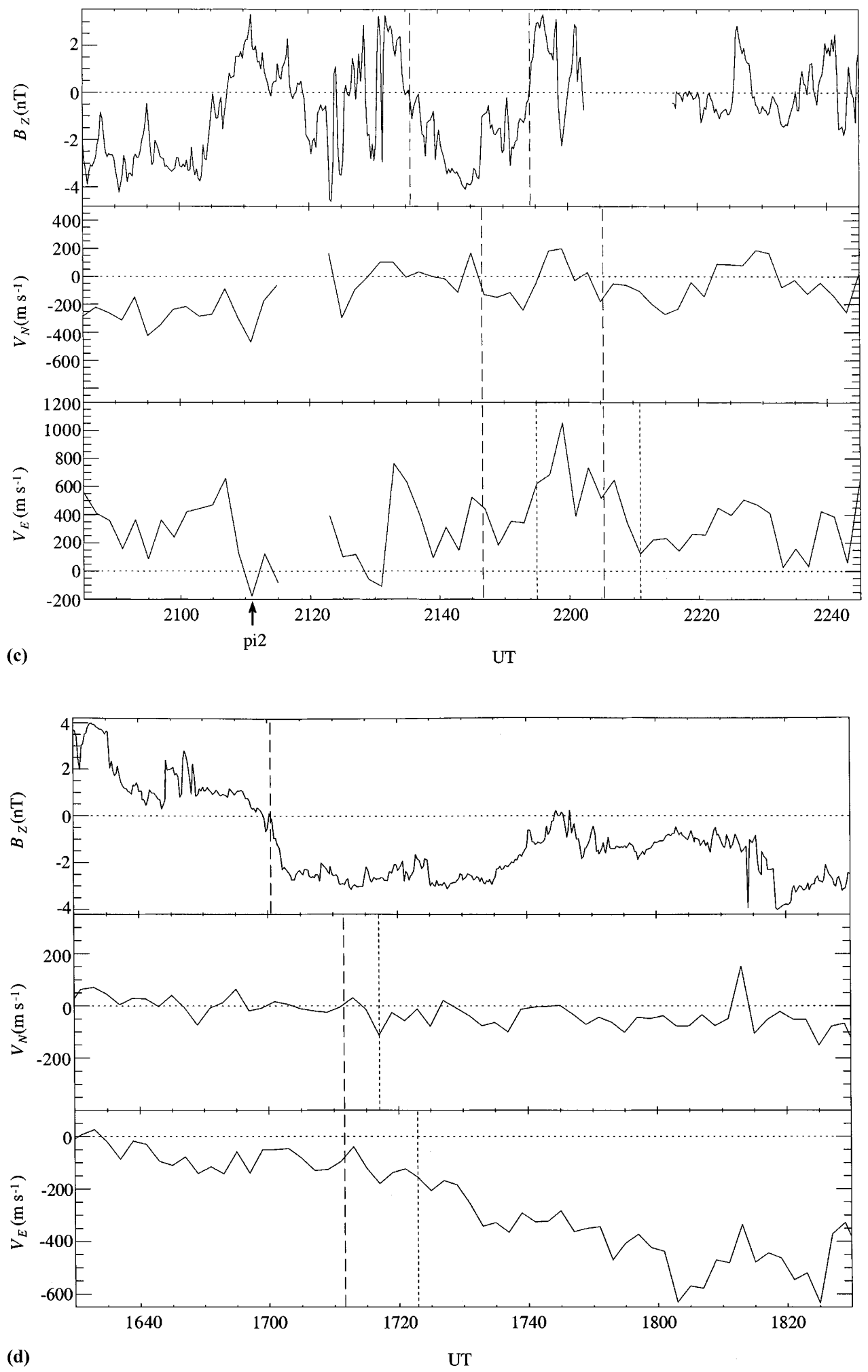

Fig. 6. c As for Fig. 2, except for the interval 2045-2245 UT (23150115 MLT) on 15 March, 1994
Fig. 6. d As for Fig. 2, except for the interval 1630-1830 UT (19002100 MLT) on 18 June, 1996 $\sim 15$ min surge in eastward flow centred near 2200 UT which appears to relate to an interval of southward IMF of similar duration centred near 2145 UT. Here the response delay after the southward turning is timed at $8 \mathrm{~min}$, while that after the northward turning is $6 \mathrm{~min}$. These values certainly lie within the range of those determined from the cross-correlation analysis in this
MLT sector, as shown previously in Fig. 5. We note that when considering the nightside flow data, magnetic records from the IMAGE and SAMNET magnetometer chains in the Scandinavian sector were examined in order to ensure that flow variations associated with substorms were not misidentified as IMF-related flow changes. During this interval, for example, a Pi2 signal 
was observed by stations of the SAMNET chain starting at $\sim 2110$ UT (indicated by the arrow in Fig. 6c), well before the flow enhancement discussed, but no clear substorm-associated onset was related to the flow enhancement itself.

Finally, in Fig. 6d we show results of a somewhat different character observed in the nightside dusk cell in the interval 1630-1830 UT (1900-2100 MLT) on 18 June, 1996. In this interval a north to south switch in IMF $B_{z}$ took place at 1701 UT (dashed line in the upper panel), following which (apart from brief intervals) a southward field of $\sim-1$ to $-3 \mathrm{nT}$ then endured for more than $1.5 \mathrm{~h}$. Thus not only the initial response of the flow can be discerned, but also its long-term behaviour under essentially continuous negative IMF $B_{z}$. The first effects of the southward turn are estimated to have arrived at the ionosphere at 1712 UT (dashed line in the lower panel), following which a weak equatorward flow and enhanced westward flow developed. The weak $\left(\sim 50-100 \mathrm{~m} \mathrm{~s}^{-1}\right)$ equatorward flows are present at and after 1717 UT (dotted line in the middle panel), with a delay of $5 \mathrm{~min}$, while the westward flow developed more gradually over a $\sim 40$ min interval, with a less well-defined onset. The time beyond which this flow development is unquestionably in progress has been set at 1723 UT as marked by the dotted line in the lower panel, with a delay of $11 \mathrm{~min}$, though it could be argued as having started earlier, at the time of excitation of the equatorward flow. Significant further development of the westward flow, from $\sim 100$ to $\sim 500 \mathrm{~m} \mathrm{~s}^{-1}$, occurred gradually after this time, presumably related to the expansion of the region of open flux and flow cells during this interval. It may be remembered that the cross-correlation results for this MLT sector, shown in Fig. 4f, indicate peak correlations of the east-west flow with a lag of $34 \mathrm{~min}$, with westward flows occurring for positive and small negative IMF $B_{z}$ reversing to become eastward for large negative IMF $B_{z}$. The event shown here in Fig. $6 \mathrm{~d}$ indicates that the response of the eastwest flow in this sector to the onset of relatively weak negative IMF $B_{z}$ is a westward flow with a prompt onset (a delay of only $\sim 5-10 \mathrm{~min}$ ) which then develops in magnitude over a few tens of minutes, thus supporting our previous suggestion that the long cross-correlation delays found for $v_{E}$ in this MLT sector are related to the time scale for the expansion of the flow cells rather than to the initial excitation of flow. In the present example, however, the flow did not subsequently reverse to eastward as in the cross-correlation results, presumably because IMF $B_{z}$ was not sufficiently negative for a sufficient interval. The flow speed of the solar wind was rather low during this interval, $V \approx 390 \mathrm{~km} \mathrm{~s}^{-1}$, such that with IMF $B_{z}$ typically $\sim-2 \mathrm{nT}$ we have $(V / 500)^{2} B_{z} \approx-1 \mathrm{nT}$. It is then noted from Fig. $4 \mathrm{f}$ that eastward flows are typically present in this MLT sector only for $(V / 500)^{2} B_{z}$ less than $\sim-4 \mathrm{nT}$. On the other hand, the rapid $(5 \mathrm{~min})$ response of the north-south component of the flow seen in Fig. 6d is compatible with the results of the cross-correlation analysis for this flow component (Fig. 4f), which shows a broad peak near zero lag with an "error bar" spanning the range from -4 to $+10 \mathrm{~min}$.

Having thus illustrated the method employed, which as we have seen is not without its ambiguities on occasion, in Fig. 7 we present the overall event analysis results, where we plot the flow response delay versus MLT in a similar format to Fig. 5. Results for the eastwest flow component are shown in Fig. 7a, where solid symbols indicate response delays determined from flow enhancements following southward turns of the IMF, while large open symbols indicate delays determined from flow decreases following northward turns. For purposes of easy comparison, these are overlaid on the cross-correlation results from the previous section, shown by the smaller open symbols with error bars joined by the dashed line. As in Fig. 5, all circular symbols indicate the excitation of eastward flow for negative IMF $B_{z}$, usually corresponding to the dawn cell, while square symbols indicate that the excitation of westward flow for negative IMF $B_{z}$, usually corresponding to the dusk cell. Several points are worth noting. First, no distinction is apparent between the response delays determined from southward as opposed to northward turns of the field. Thus the propagation of information through the ionosphere concerning the onset of flow following a southward turn of the IMF appears to occur on essentially the same time scale as the propagation of the start of the decay following a northward turn. Second, the event analysis results are in overall agreement with the cross-correlation results, such that the values determined in a given MLT sector generally fall within the "error bars" of the crosscorrelation values in that sector. The main exception occurs in the immediate post-dusk region, where, as we stated in the previous section, the "event" delays are typically short ( 5-15 $\mathrm{min})$ and comparable to results in the adjacent sectors, while the cross-correlation delays are much longer $(\sim 35 \mathrm{~min})$. The origins of this effect have already been discussed. It is also noticeable, however, that the "event" delays are typically a few minutes longer than the cross-correlation delays in the pre-noon east-west flow reversal region spanning 0800 1100 MLT. The origins of this difference are not obvious. Third, the results again show a minimum delay occurring in the immediate post-noon hours at $\sim 1400$ MLT, with a trend towards larger delays at night compared with the dayside. The average delay time for the dayside is $5.4 \pm 0.5 \mathrm{~min}$ (Table 1), compared with $3.1 \pm 0.8 \mathrm{~min}$ from the corresponding crosscorrelation analysis. For the nightside, the average delay time is $11.6 \pm 1.4 \mathrm{~min}$ (Table 1), compared with $8.0 \pm 2.4 \mathrm{~min}$ from the cross-correlation analysis. The event analysis delay times for $v_{E}$ are thus in reasonable accord with the cross-correlation delay times, though typically longer by $\sim 2-3$ min (corresponding to one or two flow data points).

Results for the north-south component are shown in Fig. $7 \mathrm{~b}$ in a similar format to Fig. 7a, except that circular symbols indicate equatorward flow for negative IMF $B_{z}$, while square symbols indicate poleward flow (as in Fig. 5b). The average response delay determined 
from the dayside data is $5.8 \pm 0.5 \mathrm{~min}$, again comparable with the corresponding dayside cross-correlation result of $3.7 \pm 1.8 \mathrm{~min}$. The number of nightside determinations and their distribution in MLT is insufficient to allow a meaningful average to be taken.

As with the cross-correlation results, we have also combined the east-west and north-south flow "event" data together, by taking the true response delay to correspond to the first response observed in the flow irrespective of the component in which it was observed. In Fig. 7c we therefore show this minimum delay time versus MLT for all 69 events analysed in this study. The closed symbols represent results obtained from flow enhancements following southward turns of the IMF (46 events), while the open symbols represent results from the onset of flow decays following northward turns of the IMF (23 events). As before, no systematic differences are observed between these cases. Similarly, square symbols indicate that the first response was seen in the east-west flow component (38 events), circular symbols that the first response was seen in the northsouth flow component (18 events), and triangles indicate simultaneous responses in both components (13 events). No clear MLT trends are observed in these types of response. However, evidence is again obtained of increasing delays from dayside to nightside, with minimum delays in the post-noon sector. The average delay for the dayside data is $4.8 \pm 0.5 \mathrm{~min}$, while the average for the nightside data is $8.7 \pm 1.1 \mathrm{~min}$ (Table 1). Noting the post-noon minimum, however, if instead we average over the 12-h sector centred on 1400 MLT we find a value of $4.8 \pm 0.5 \mathrm{~min}$ as before, while if we average over the 12-h sector centred on 0200 MLT we find a value of $9.2 \pm 0.8 \mathrm{~min}$ (Table 1). To illustrate this difference directly, Fig. 8 shows normalised histograms of the distribution of delay times for the 12-h
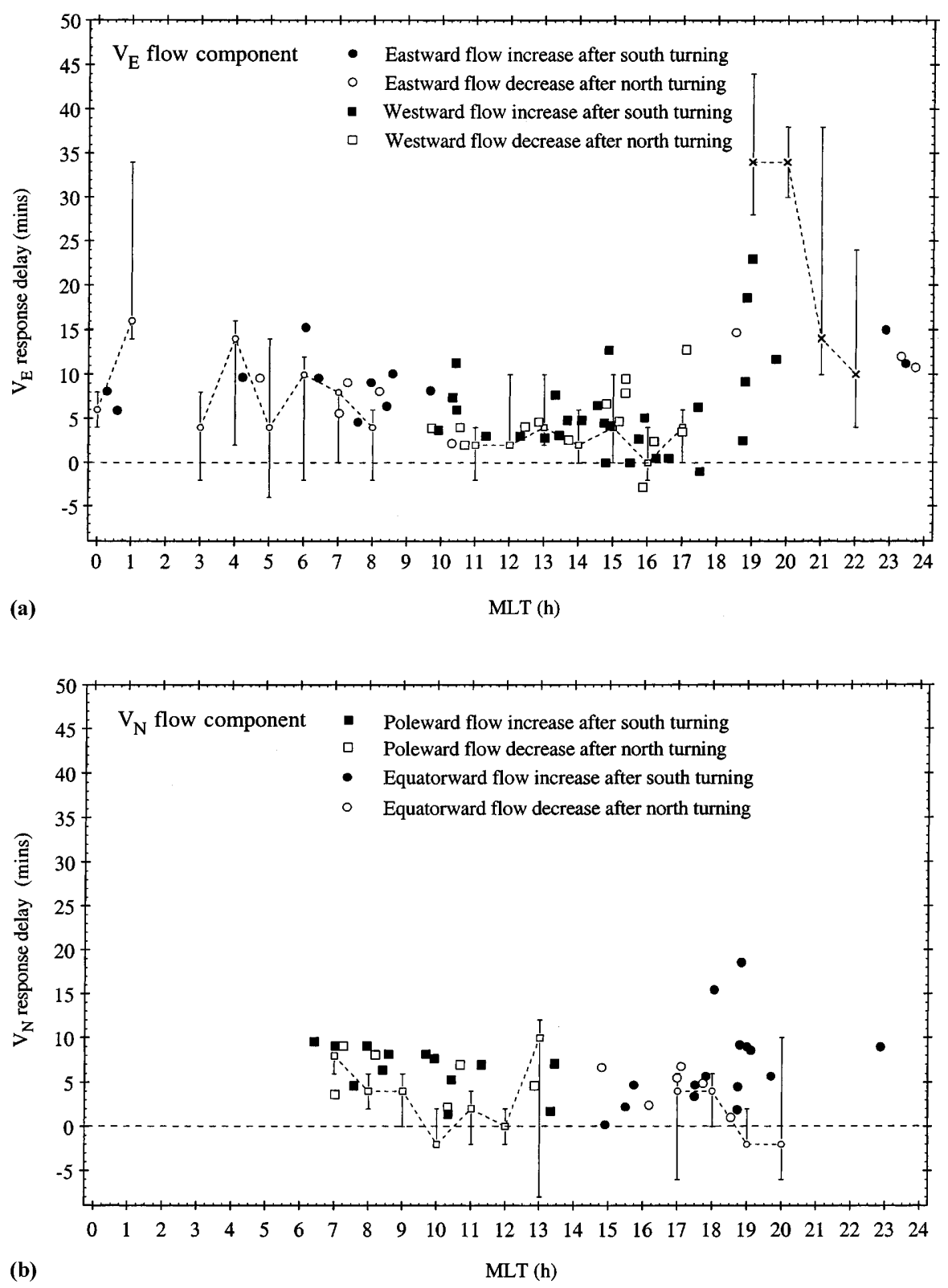

Fig. 7. a Ionospheric flow response delays versus MLT determined from the event analysis keyed to sudden changes in the sense of the north-south component of the IMF, for the east-west component of flow. Solid symbols indicate delay times determined from the onset of increases in flow associated with southward turns of the IMF, while open symbols indicate response delays determined from the onset of flow decay associated with northward turns of the IMF. Circular symbols indicate that the flow excited is eastward, generally corresponding to the dawn flow cell, while squares indicate the flow is westward, generally corresponding to the dusk flow cell (as in Fig. 5). These data are plotted over the delay times determined from the $v_{E}$ cross-correlation analysis as in Fig. 5 (smaller open symbols with error bars joined by dashed lines)

Fig. 7. b As for a, except for the northsouth flow component. The square symbols indicate poleward flow, while the circular symbols indicate equatorward flow (as in Fig. 5) 


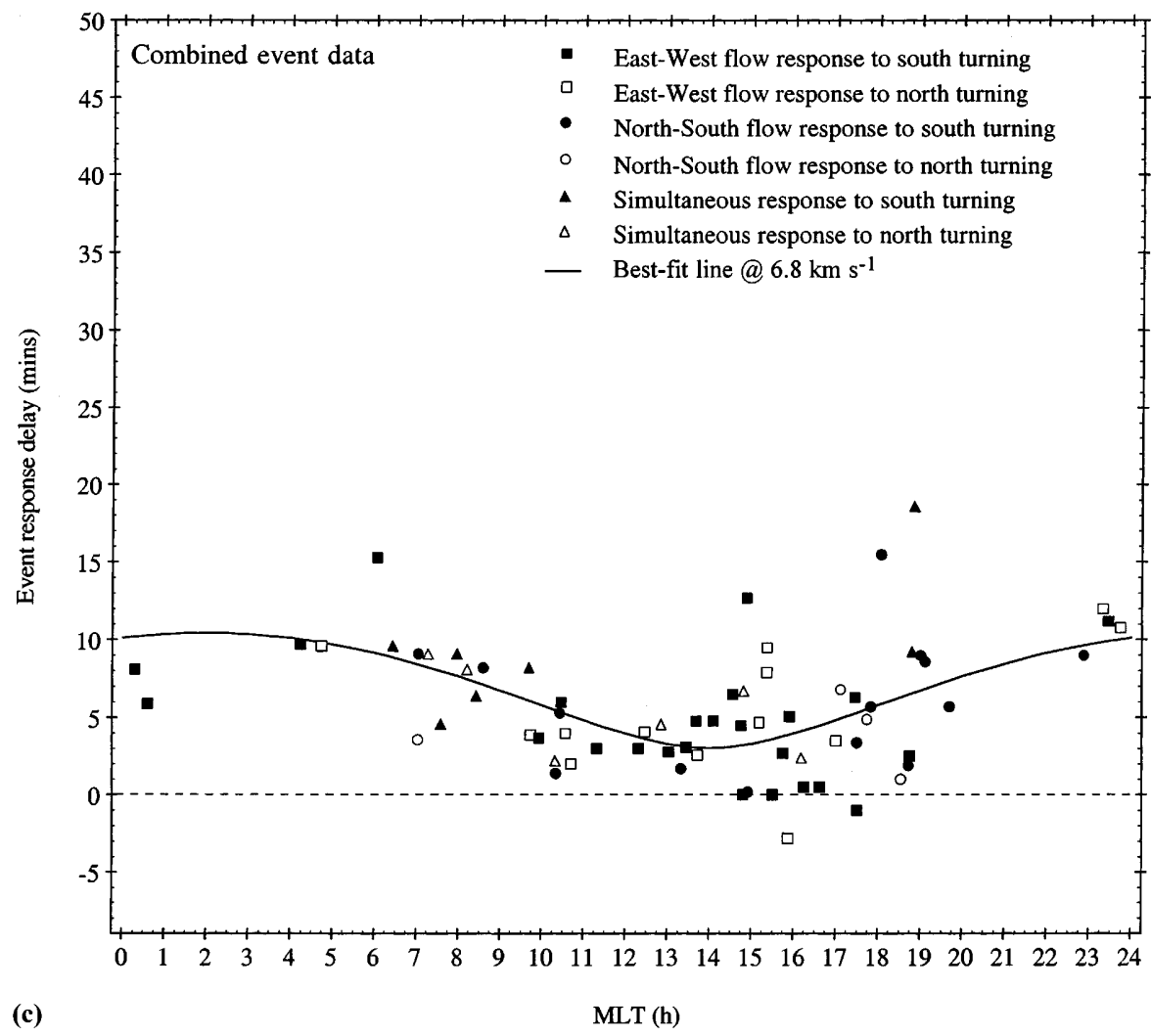

Fig. 7. c As for a, except that the minimum delay is shown for each event, irrespective of which flow component is involved. Closed symbols indicate responses to southward turns of the IMF, while open symbols indicate responses to northward turns of the IMF. Responses observed first in the east-west flow component are shown by squares, while those observed first in the north-south component are shown by circles. If both components responded simultaneously the symbol is plotted as a triangle. These data have been least-squares fit to the same simple theoretical model as in Fig. 5c, shown by the solid line. In this case the best-fit epicentre of information propagation was also found to lie at 1400 MLT, while the expansion phase speed was found to be $6.8 \mathrm{~km} \mathrm{~s}^{-1}$ sector centred on 1400 MLT (Fig. 8a), and for the 12-h sector centred on 0200 MLT (Fig. 8b). The data are shown in 2-min bins, normalised to the total number of events in the two regions. The average values given above are indicated by the arrow and dot-dashed line. The two distributions are clearly different, with the nightside values being shifted to longer delay times compared with the dayside values. Even so, it should again be emphasised that the delay times are found to be relatively short at all local times, with the difference between the 1400 MLT sector and 0200 MLT sector average values being only $4.4 \pm 0.9 \mathrm{~min}$.

The results presented here thus again provide evidence of a propagation of information about changes in the flow through the ionosphere from the dayside to the nightside, with minimum delays in the immediate postnoon sector. We have again fit this data to the simple theoretical model of information propagation that we employed above for the combined cross-correlation results (Fig. 5c), and have derived the best fit local time of the epicentre from which the propagation starts (assumed to lie at $77^{\circ}$ magnetic latitude), and the phase speed. This procedure yields an epicentre located at 1400 MLT, and a phase speed of $6.8 \mathrm{~km} \mathrm{~s}^{-1}$. The bestfit line is plotted in Fig. 7c, and is seen to follow the trends in the event data reasonably well.

\section{Summary and discussion}

We have compared $\sim 300$ simultaneous hours of observations of the high-latitude ionospheric flow obtained by the EISCAT UHF radar with measurements of the interplanetary field made by the IMP-8 spacecraft upstream from the Earth's magnetosphere, in order to determine the time scale on which the ionospheric flow responds to changes in the north-south component of the IMF. The field-perpendicular ionospheric flow vectors were determined using tristatic velocity data from the EISCAT CP-1-K common programme experiment, in which the plasma velocity is continuously monitored with 2-min resolution at an altitude of $278 \mathrm{~km}$ along the field line at Tromsø $\left(66.3^{\circ}\right.$ magnetic latitude). The data cover all magnetic local times, though with a preference for the hemisphere centred on dusk compared with the hemisphere centred on dawn. Two analysis techniques have been employed. In the first the flow data were divided into overlapping 2-h intervals of MLT, and cross-correlated with the halfwave rectifier function $V^{2} B_{s}$, where the latter data were suitably shifted in time to take account of the propagation of the IMF from the spacecraft to the subsolar magnetopause, and its effects from thence to the cusp ionosphere. A similar cross-correlation analysis was previously performed by Etemadi et al. (1988) using data from the EISCAT "Polar" experiment (at latitudes $\sim 71^{\circ}-73^{\circ}$ ), but the analysis only spanned the dayside hours between $\sim 1030$ and $\sim 1700$ MLT, corresponding mainly to the westward flows of the "dusk" flow cell. Here we have extended these results to include the dayside dawn flow cell and the nightside hours.

Our cross-correlation results show that for the eastwest flow component statistically significant peaks in the cross-correlation coefficient are obtained at nearly all 

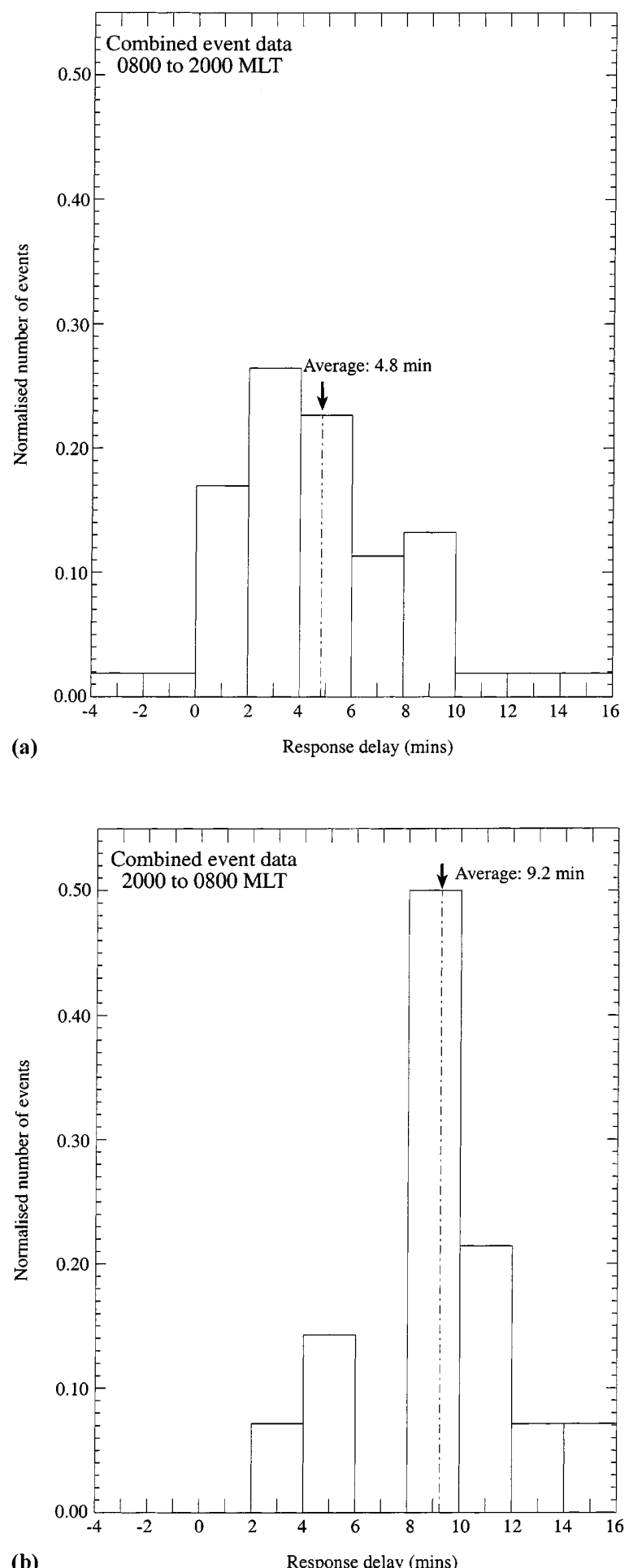

(b)

Fig. 8. a Histogram showing the normalised distribution of the number of events contributing to each 2-min bin of ionospheric flow delay time, for the $12-\mathrm{h}$ local time sector centred on 1400 MLT. The average delay of all the dayside events is marked by the arrow and dotdashed line. b As for a except for the 12-h local time sector centred on 0200 MLT

local times, except principally for the pre-noon hours ( 0900-1000 MLT) where the east-west flow reverses in sense between the flow cells. However, the nightside cross-correlation coefficients are typically smaller than on the dayside and the results correspondingly more scattered. The scatter-plots of the east-west flow versus $V^{2} B_{s}$ corresponding to the peak cross-correlation coefficient generally show relatively weak averaged flows for northward IMF which change monotonically as $V^{2} B_{s}$ becomes increasingly negative. In this case we may interpret the lag of the peak correlation as the IMFmodulated flow excitation and decay delay time, which lies typically in the range $0-15 \mathrm{~min}$. However, in the premidnight Harang region the response times become significantly longer ( $\sim 30-40 \mathrm{~min})$, while the corresponding scatter plots for the east-west flow show the presence of non-monotonic behaviour. Westward flows which are present for positive IMF $B_{z}$ intensify for small southward IMF, and then decline and reverse to become eastward for strong negative IMF $B_{z}$. In this case the extremum in the cross-correlation coefficient need not occur at the excitation and decay delay time, but at a later time more representative of the time scale for flow reversal relative to the northward IMF "baseline". Such data have been excluded from our subsequent analysis.

The average east-west flow response delay time for the dayside cross-correlation data, weighted according to the inverse of the uncertainty estimates of the values, is found to be $3.1 \pm 0.8 \mathrm{~min}$. This value is closely comparable to the corresponding results of Etemadi et al. (1988) when the $\sim 2$ min propagation from the magnetopause to the ionosphere is (as here) subtracted. On the nightside, the weighted average value increases to $8.0 \pm 2.4 \mathrm{~min}$, where the results from the 1900-2000 MLT Harang region have been omitted. Statistically significant responses of the north-south flow are more restricted in MLT, and are confined to the dayside interval 0700-1300 MLT for poleward flows excited by southward IMF, and to the dusk sector 1700-2000 MLT for equatorward flows excited by southward IMF. The weighted average response delay of the former values is $3.7 \pm 1.8 \mathrm{~min}$, entirely compatible with the dayside results for the east-west flow. The dusk-sector values are also consistent with a relatively small delay in the range 0-5 min, though the cross-correlation distributions are typically very broad and the error bars correspondingly large.

When these data are suitably combined together they indicate that the minimum response delay occurs at $\sim 1400 \mathrm{MLT}$. The overall weighted average delay for the 12 -h sector centred on 1400 MLT is $1.3 \pm 0.8 \mathrm{~min}$, while that for the 12-h sector centred on 0200 MLT is $8.8 \pm 1.7 \mathrm{~min}$. The implication is that information about IMF-related modulations of the flow propagates through the ionosphere away from the postnoon sector. In order to quantify the phase speed of that propagation we have fitted a simple model to the combined data in which information propagates radially outwards from an epicentre located on the dayside open-closed field line boundary (taken to lie at $77^{\circ}$ magnetic latitude) at a constant phase speed. In this case the best fit is obtained for an epicentre centred at 1400 MLT and a phase speed of $9.0 \mathrm{~km} \mathrm{~s}^{-1}$.

In the second complementary analysis of the same data set we have inspected the IMF time series for sharp 
changes in the sign of the north-south component, and have then examined the ionospheric measurements to determine the time at which the flow responds, either with an increase in flow following a southward turn, or a decrease in flow following a northward turn. In all, a total of 69 such events have been analysed and included in the study. A similar analysis was previously performed on the EISCAT "Polar" data by Todd et al. (1988), but included many fewer events and was again restricted in local time coverage mainly to the dayside hours. The results confirm and extend those derived from the crosscorrelation study summarised. For the east-west flow component, minimum delays are centred on the postnoon sector, with average response delays of $5.4 \pm$ $0.5 \mathrm{~min}$ on the dayside, increasing to $11.6 \pm 1.4 \mathrm{~min}$ on the nightside. These values are consistent with, but a little longer (by $\sim 2-3 \mathrm{~min}$ ) than the cross-correlation values for the east-west flow quoted. No distinction was apparent between the response times determined from the onset of flow excitation following southward turns, and the onset of flow decay following northward turns. For the north-south flow component, the dayside average was found to be $5.8 \pm 0.5 \mathrm{~min}$, with insufficient event determinations being available on the nightside to take a meaningful average.

We have also combined the event data for both flow components together, and have taken the flow response delay to correspond to the first flow change observed in each event, irrespective of which component is involved. These data again show a minimum delay in the postnoon sector, with increased response delays on the nightside. When the simple theoretical model outlined is fitted to this data, it is found that the best fit is again obtained for an epicentre of information propagation centred on 1400 MLT, with a phase speed of $6.8 \mathrm{~km} \mathrm{~s}^{-1}$. The average of the delays for the 12 -h sector centred on 1400 MLT was found to be $4.8 \pm 0.5 \mathrm{~min}$, increasing to $9.2 \pm 0.8 \mathrm{~min}$ for the $12-\mathrm{h}$ interval centred on 0200 MLT.

It may be noted that the post-noon minimum in the response delay found here was also observed in the earlier studies by Etemadi et al. (1988) and Todd et al. (1988). This asymmetry may possibly reflect the Parker spiral structure of the IMF, which should generally produce a "first contact" of a similarly oriented interplanetary tangential discontinuity with the post-noon magnetopause. Examination of our interplanetary data shows that the plane of the discontinuities were indeed preferentially oriented in this direction. The phase speed of propagation is also consistent with the values deduced from the earlier, more limited, studies by Etemadi et al. (1988), Todd et al. (1988), Lockwood et al. (1993), and Saunders et al. (1992), whose data are consistent with values in the range $5-10 \mathrm{~km} \mathrm{~s}^{-1}$. The short (several minute) response delays found here are also consistent with the overall delays of $8 \pm 8 \mathrm{~min}$ found in the study presented by Ridley et al. (1998). However, the latter authors also interpreted their data as indicating an essentially simultaneous response (within $\sim 1 \mathrm{~min}$ ) at all local times over the high-latitude region. This conclusion is not in accord with our finding here of longer response times on the nightside than on the dayside, though we would point out that the difference we found here between these responses is only $\sim 6$ min. Finally we note that our response times are much shorter than the values of $\sim 20-30 \mathrm{~min}$ determined by Hairston and Heelis (1995) using DMSP spacecraft data. We thus concur with these authors that the response times which they deduced are more characteristic of the development of the flow pattern as a whole following changes in the IMF, rather than the IMF-modulated flow excitation and decay delay which has been our primary concern here.

Acknowledgements. The authors would like to thank the Director of EISCAT and his staff for operating the facility and providing the data. EISCAT is an international association supported by the research councils of Finland (SA), France (CNRS), Germany (MPG), Japan (NIPR), Norway (NAVF), Sweden (NFA), and the United Kingdom (PPARC). The IMP-8 magnetometer data (Principal Investigator: R.P Lepping) was provided by the National Space Science Data Centre, while the fine resolution plasma data (Principal Investigator: A.J Lazarus) was provided by the Massachusetts Institute of Technology. We extend special thanks to Dr J.A Davies for support with the EISCAT data and constructive discussion throughout the work. We also thank William Cowley for help with the event analysis during a work experience placement at the University of Leicester, and the referees for thoughtful and provocative comment on earlier drafts. This work was performed while one of us (HK) was supported by a studentship provided by the University of Leicester.

Editor-in-chief D. Alcayde' thanks M.R. Haviston and another referee for their help evaluating in this paper.

\section{Appendix}

In order to accurately determine the response time of the ionospheric flow to changes in the direction of the IMF observed by a spacecraft upstream of the bow shock, a careful estimate must be made of the delay between an IMF feature being observed by the spacecraft and the arrival of the first possible effect at the ionosphere. This propagation delay consists of the sum of three components: (1) the time between the appearance of the feature at the spacecraft and its arrival at the subsolar bow shock, (2) the frozen-in transit time across the subsolar magnetosheath, and (3) the Alfvénic propagation time along open field lines from the subsolar magnetopause to the cusp ionosphere. The method used to determine each of these contributions will now be discussed, together with their application in the case of the present study and their likely uncertainties.

\section{Transit time between the spacecraft and subsolar bow shock}

For a spacecraft located on the Earth-Sun line, the transit time of field changes between the spacecraft and the subsolar bow shock is simply determined from the distance to the bow shock (determined from empirical models as described below) divided by the observed solar wind speed. However, in general the spacecraft will be located at some distance from the Earth-Sun line, and 
we then require to know where corresponding field changes are located on the Earth-Sun line at the same time as they are observed by the spacecraft. In other words we need to determine, or assume, the orientation of the "phase fronts" in the IMF so that the intersection with the Earth-Sun line of the phase front which passes through the satellite can be determined. We therefore examined the IMF data corresponding to each 2-h interval of radar data, and determined the orientation of the plane of the sharp field changes which were observed, assuming that these changes represent tangential discontinuities propagating in the solar wind. Typically the field vectors were averaged over 10-15 min intervals on either side of the discontinuity, and their cross-product taken to determine the discontinuity normal. Usually, several (2 to 6) such determinations could be made for each 2-h interval, and in these cases the orientations of the discontinuities were generally found to be mutually consistent, with normals typically lying near the equatorial plane perpendicular to the garden-hose direction. These were then used to determine an overall representative delay time for the interval according to the algorithm described later. In a few cases, however, no sharp field changes occurred in the interval in question, and in these cases we simply assumed that the phase fronts were aligned perpendicular to the Earth-Sun line.

\section{Estimation of the subsolar magnetosheath transit time from the bow shock to the magnetopause}

When the solar wind encounters the subsolar bow shock its speed is suddenly reduced by a factor of $\sim 4$ for large upstream magnetosonic Mach numbers. According to gas-dynamic models the speed then continues to fall as the plasma traverses the magnetosheath and reaches the magnetopause. Accurate estimation of the overall propagation time requires that these effects are taken into account. In order to do this we need to know the geocentric distances of the magnetopause and bow shock, and to model the plasma speed between them. Roelof and Sibeck (1993) have provided a comprehensive empirical model of the position of the magnetopause, in which the boundary is modelled as an ellipsoid of revolution about the aberrated $\mathrm{X}$ axis whose form depends on both IMF $B_{z}$ and the solar wind dynamic pressure $p$. For purposes of our study, we wish to determine one representative delay time that can be applied to few-hour segments of interplanetary data. Since IMF $B_{z}$ typically varies significantly about a nearzero mean on such time scales, we simply employ the Roelof and Sibeck (1993) model subsolar magnetopause position for IMF $B_{z} \approx 0 \mathrm{nT}$ which is valid for a typical dynamic pressure of $2 \mathrm{nPa}$ (a value of $\sim 10.8 \mathrm{R}_{\mathrm{E}}$ ), and then scale this value according to the usual one sixth power law of $p$. The parameterisation of the subsolar magnetopause position $R_{M P}$ thus employed is

$$
R_{M P}=\frac{12.1}{p(\mathrm{nPa})^{1 / 6}}=\frac{111}{\left[n\left(\mathrm{~cm}^{-3}\right) V^{2}\left(\mathrm{~km} \mathrm{~s}^{-1}\right)\right]^{1 / 6}} \mathrm{R}_{\mathrm{E}} .
$$

This formula is in good overall accord with the Roelof and Sibeck model for $\left|B_{z}\right|$ not too large, and is also in reasonable agreement with the model results of Petrinec and Russell (1993).

Using this value, the distance to the subsolar bow shock can then be determined, using the results of the empirical study by Peredo et al. (1995). From a comprehensive analysis of observed shock locations these authors found that the subsolar shock is typically located at a distance which is larger than that of the subsolar magnetopause by a factor of 1.46 . We thus parametrise the subsolar shock location as

$R_{B S}=\frac{17.6}{[p(\mathrm{nPa})]^{1 / 6}}=\frac{162}{\left[n\left(\mathrm{~cm}^{-3}\right) V^{2}\left(\mathrm{~km} \mathrm{~s}^{-1}\right)\right]^{1 / 6}} \mathrm{R}_{\mathrm{E}}$.

We now require a model of the flow speed between the subsolar shock and magnetopause, and simply take a linear variation from a value of $V_{B S}$ just downstream from the shock and $V_{M P}$ at the magnetopause. Integration of $(d s / V)$ between $R_{B S}$ and $R_{M P}$ then yields the frozen-in propagation time across the subsolar magnetosheath as

$\tau_{s h}=\frac{\left(R_{B S}-R_{M P}\right)}{V_{B S}-V_{M P}} \ln \left(\frac{V_{B S}}{V_{M P}}\right)=\frac{0.46 R_{M P}}{V_{B S}-V_{M P}} \ln \frac{V_{B S}}{V_{M P}}$.

We finally require values for $V_{B S}$ and $V_{M P}$. The usual shock jump conditions determine $V_{B S}$ in terms of the upstream speed $V_{S W}$ as

$\frac{V_{B S}}{V_{S W}}=\frac{(\gamma-1) M^{2}+2}{(\gamma+1) M^{2}}$,

where $M$ is the magnetosonic Mach number and $\gamma=5 / 3$. Substituting an average value of $M=5.3$ (Peredo et al., $1995)$ into Eq. (4) gives $\left(V_{B S} / V_{S W}\right) \approx 0.28$, and this is the value for $V_{B S}$ which we adopt here. The reconnectionassociated velocity of inflow at the magnetopause, $V_{M P}$, must be taken to be a finite value to avoid the logarithmic singularity in Eq. (3), and we assume a nominal value of $20 \mathrm{~km} \mathrm{~s}^{-1}$. Taken together with reasonable values for the magnetosheath field at the magnetopause of a few tens of nT, and reasonable lengths for the reconnection line of several $R_{E}$, this value implies reasonable reconnection-driven convection voltages of several tens of $\mathrm{kV}$. However, our results are not sensitive to the exact choice. Substituting these values into Eq. (3) finally yields

$\tau_{s h}=\frac{1.66 R_{M P}}{\left(V_{S W}-72\right)} \ln \left(\frac{V_{S W}}{72}\right)$,

where $V_{S W}$ is in $\mathrm{km} \mathrm{s}^{-1}$, and $R_{M P}$ is in $\mathrm{km}$, determined from Eq. (1) with $\mathrm{R}_{\mathrm{E}}=6375 \mathrm{~km}$. Thus $\tau_{s h}$ is determined directly in terms of the density and velocity of the upstream solar wind plasma.

We finally note that if Eq. (5) is interpreted as the magnetosheath thickness of $0.46 R_{M P}$ divided by an "average" magnetosheath speed $\left\langle V_{s h}\right\rangle$, then for typical values of $V_{S W} \sim 500 \mathrm{~km} \mathrm{~s}^{-1}$ we find $\left\langle V_{s h}\right\rangle \approx\left(V_{S W} / 8\right)$. Our formula therefore produces a final value which is 
very similar to that obtained by simply dividing the subsolar magnetosheath thickness by an average magnetosheath speed which assumes a jump by a factor of 4 at the shock and a linear decrease to zero at the magnetopause, as employed in previous studies (e.g. Lester et al., 1993).

\section{Alfvénic propagation from the magnetopause to the cusp ionosphere}

Once reconnection occurs at the dayside magnetopause, its effects on the flow are transmitted along the newlyopened field lines by an Alfvén wave. The transit time from the subsolar magnetopause to the ionosphere can then be determined as approximately one quarter of the period of fundamental mode eigenoscillations of the outer dayside field lines. Since these periods are typically found to lie in the range 5-10 min (e.g. Poulter et al., 1984), we have taken the magnetopause-to-ionosphere transit time to be $2 \mathrm{~min}$.

\section{Application to the present study}

We finally describe how the methods outlined have been applied in the present study. For purposes of the cross-correlation analysis we require one representative IMF delay time corresponding to 2-h segments of radar data. To obtain this delay we first scanned the corresponding IMF data for sharp field changes to determine the orientation of the phase fronts in the field, as described. Using each orientation so determined, we then calculated the total delay time as a function of UT over the 2-h interval, using the measured solar wind density and velocity values to determine the subsolar shock position according to Eq. (2) and the magnetosheath transit time from Eqs. (1) and (5). The "delay" time series was then averaged over the whole interval to provide a single averaged delay time determined from that phase front orientation (typically the variation of the delay time over such intervals is $\sim 1 \mathrm{~min}$ or less). This procedure was then repeated for each phase front orientation determined during the interval (typically 2-6), yielding a set of averaged delay times. Normally these proved to be mutually consistent within 1-2 min. A representative delay time value was then chosen for the interval, and 2 min-averaged IMF vectors were derived from the $15 \mathrm{~s}$ values, corresponding to the 2 min averaged radar values with the representative propagation delay removed. These averaged IMF values were then used for the cross-correlation analysis. Essentially the same procedures were used for the event study, except that the orientation of the phase front of the particular IMF "event" in question was used as the basis of the averaged delay time estimate, and the average was taken over the same 10-15 min intervals on either side of the discontinuity as were used to determine the direction of the discontinuity normal.

\section{Uncertainty estimates}

We finally consider the likely level of the random and systematic errors in our propagation delay determinations. The first source of uncertainty arises from our determinations of the IMF "phase front" orientations in the interplanetary medium, together with the variations of this orientation and of the speed of the solar wind over the $\sim 2 \mathrm{~h}$ intervals employed in this study. As indicated, changes in the solar wind speed over such intervals typically produce variations in the estimated propagation delay of $\sim 1 \mathrm{~min}$ or less for a fixed "phase front" orientation, and analysis of several "phase fronts" in a particular interval generally provide consistent results within $\sim 1-2 \mathrm{~min}$. These uncertainties contribute to a random error in the propagation delay values which we thus estimate to be about $\pm 1 \mathrm{~min}$. This estimate is somewhat smaller than would be inferred from the recent results of Ridley et al. (1998) and Collier et al. (1998), both of whom undertook IMF propagation studies between spacecraft widely separated in the solar wind (in particular WIND and IMP-8). The random timing errors determined in these studies are of order $\pm d_{\text {perp }} / V_{S W}$, where $d_{\text {perp }}$ is the separation of the observing points in the plane perpendicular to the Earth-Sun line. The corresponding distance in our case is the perpendicular distance of IMP-8 from the EarthSun line, which we may take typically to be $\sim 20 \mathrm{R}_{\mathrm{E}}$. The uncertainty in the spacecraft to subsolar bow shock propagation time would then be $\sim \pm 4 \mathrm{~min}$ in values which range typically between 2 and $8 \mathrm{~min}$, compared with the $\pm 1 \mathrm{~min}$ which we estimated on the basis described earlier. The meaning of an error of order $\pm d_{\text {perp }} / V_{S W}$ is that the orientation of the "phase fronts" of the IMF variations is essentially undetermined; its literal meaning is that the normal may lie anywhere within a cone of angles $\pm 45^{\circ}$ about the Earth-Sun line. However, our analysis indicates that the "phase fronts" associated with the large changes in IMF $B_{z}$ which are of special significance in this study are rather more organised in direction than this, and lie typically near the equatorial plane orthogonal to the garden-hose direction, as previously indicated. Thus when several discontinuities are analysed in a particular interval, the variation in "phase front" orientation typically produces a variation in the estimated propagation time of $\sim \pm 1 \mathrm{~min}$ as indicated, rather than the $\sim \pm 4 \mathrm{~min}$ that would be expected on the basis of the analysis of more general variations in the IMF presented by Collier et al. (1998). For the circumstances of the present study, therefore, the smaller value appears more appropriate.

The second source of uncertainty lies in the modelled positions of the bow shock and magnetopause, and more particularly the distance between them, and in the magnetosheath flow model employed. As indicated, the models of the bow shock and magnetopause positions we have employed are based on the results of recent statistical studies incorporating large numbers of positional observations, while the model of the subsolar magnetosheath flow is based on shock jump conditions and the results of gas-dynamic models. It therefore 
seems that large systematic uncertainties are unlikely. Typically, the thickness of the subsolar magnetosheath determined from these models is $4-5 \mathrm{R}_{\mathrm{E}}$, and judging from the scatter in the positional data in the statistical studies cited, the uncertainty in this value is probably around $10 \%$, i.e. about $\pm 0.5 \mathrm{R}_{\mathrm{E}}$. Equation (3) then yields a likely random error in the magnetosheath propagation delay of less than $\pm 1 \mathrm{~min}$. The systematic error seems unlikely to be larger than this. The third source of potential error arises from the Alfvénic propagation of information from the magnetopause to the cusp ionosphere, taken to be $2 \mathrm{~min}$ on the basis of the measured periods of ULF waves on outer dayside field lines. Based also on the variability of the latter, we estimate that the random and systematic error of this propagation time is $\pm 1 \mathrm{~min}$. Taken together, therefore, and recognising the uncorrelated nature of the separate contributions to the uncertainties involved, we estimate the random error in our calculated propagation delays to be about \pm 2 min (in a total which lies typically in the range $5-15 \mathrm{~min}$ ), while the systematic error is likely to be comparable with this or smaller. We note that our estimate of the random error is consistent with the degree of scatter observed in our results, such as that in the "event study" displayed in Fig. 7.

\section{References}

Baker, D. N., E. W. Hones Jr., J. B. Paynes, and W. C. Feldman, A high time resolution study of interplanetary parameter correlations with AE, Geophys. Res. Lett., 8, 170, 1981.

Baker, D. N., R. D. Zwikl, S. J. Bame, E. W. Hones Jr., B. T. Tsurutani, E. J. Smith, and S.-I. Akasofu, An ISEE 3 high time resolution study of interplanetary parameter correlations with magnetospheric activity, J. Geophys. Res., 88, 6230, 1983.

Bargatze, L. F., D. N. Baker, R. L. McPherron, and E. W. Hones Jr., Magnetospheric impulse response for many levels of geomagnetic activity, J. Geophys. Res., 90, 6387, 1985.

Burton, R. K., R. L. McPherron, and C. T. Russell, An empirical relationship between interplanetary conditions and $D_{s t}, J$. Geophys. Res., 90, 4204, 1975.

Clauer, C. R., R. L. McPherron, C. Searls, and M. Kivelson, Solar wind control of auroral zone geomagnetic activity, Geophys. Res. Lett., 8, 915, 1981.

Collier, M. R., J. A. Slavin, R. P. Lepping, A. Szabo, and K. Ogilvie, Timing accuracy for the simple planar propagation of magnetic field structures in the solar wind, Geophys. Res. Lett., 25, 2509, 1998.

Cowley, S. W. H., The causes of convection in the Earth's magnetosphere: a review of developments during the IMS, Rev. Geophys. Space Phys. 20, 531, 1982.

Cowley, S. W. H., and M. Lockwood, Excitation and decay of solar wind-driven flows in the magnetosphere-ionosphere system, Ann. Geophysicae, 10, 103, 1992.

Cowley, S. W. H., and M. Lockwood, Incoherent scatter radar observations related to magnetospheric dynamics, Adv. Space Res., 20, (4/5)873, 1997.

Dungey, J. W., Interplanetary field and the auroral zone, Phys. Res. Lett., 6, 47-48, 1961.

Etemadi, A., S. W. H. Cowley, M. Lockwood, B. J. I. Bromage, D. M. Willis, and H. Lühr, The dependence of high-latitude dayside ionospheric flows on N-S component of IMF: a high time resolution correlation analysis using EISCAT 'Polar' and AMPTE-UKS and -IRM data, Planet. Space Sci., 36, 471, 1988 .
Hairston, M. R., and R. A. Heelis, Response time of the polar convection pattern to changes in the north-south direction of the IMF, Geophys. Res. Lett., 22, 631, 1995.

Heppner, J. P., Polar cap electric field distributions related to the interplanetary magnetic field direction, J. Geophys. Res., 27, 4877, 1972.

Lester, M., O. de la Beaujardière, J. C. Foster, M. P. Freeman, H. Lühr, J. M. Ruohoniemi, and W. Swider, The response of the large scale ionospheric convection pattern to changes in the IMF and substorms: results from the SUNDIAL 1987 campaign, Ann. Geophysicae, 11, 556, 1993.

Lockwood, M., Solar wind-magnetosphere coupling, Proc. EISCAT Inte. School in Corsica, Ed. D. Alcaydé, 1995.

Lockwood, M., and S. W. H. Cowley, Comment on "A statistical study of the ionospheric convection response to changing interplanetary field conditions using the assimilative mapping of ionospheric electrodynamics technique" by A.J. Ridley et al., J. Geophys. Res., 104, 4387, 1999.

Lockwood, M., A. P. van Eyken, B. J. I. Bromage, D. M. Willis, and S. W. H. Cowley, Eastward propagation of a plasma convection enhancement following a southward turning of the interplanetary magnetic field, Geophys. Res. Lett., 13, 72, 1986.

Lockwood, M., J. Moen, S. W. H. Cowley, A. D. Farmer, U. P. Løvhaug, H. Lühr, and V. N. Davda, Variability of dayside convection and motions of the cusp/cleft aurora, Geophys. Res. Lett., 20, 1011, 1993.

Newell, P. T., C.-I. Meng, D. G. Sibeck, and R. Lepping, Some lowaltitude cusp dependencies on the interplanetary magnetic field, J. Geophys. Res., 94, 8921, 1989.

Nishida, A., Coherence of geomagnetic DP-2 fluctuations with interplanetary magnetic variations, J. Geophys. Res., 73, 1795, 1968.

Perreault, P., and S.-I. Akasofu, A study of geomagnetic storms, Geophys. J. R. Astron. Soc., 54, 547, 1978.

Petrinec, S. P., and C. T. Russell, External and internal influences on the size and shape of the magnetopause, Geophys. Res. Lett., 20, 339, 1993.

Peredo, M., J. A. Slavin, E. Mazur, and S. A. Curtis, Three dimensional position and shape of the bow shock and their variation with the Alfvénic, sonic and magnetosonic Mach numbers and interplanetary magnetic field orientation, J. Geophys. Res., 100, 7907, 1995.

Poulter, E. M., W. Allan, J. G. Keys, and E. Nielsen, Plasmatrough ion mass densities determined from ULF pulsation eigenperiods, Planet. Space Sci., 32, 1069, 1984.

Reiff, P. H., and J. L. Burch, IMF By-dependent plasma flow and Birkeland currents in the dayside magnetosphere. 2. A global model for northward and southward IMF, J. Geophys. Res., 90, 1595, 1985.

Reiff, P. H., R. W. Spiro, and T. W. Hill, Dependence of polar cap potential drop on interplanetary parameters, J. Geophys. Res., 86, 7639, 1981.

Ridley, A. J., G. Lu, C. R. Clauer, and V. O. Papitashvili, A statistical study of the ionospheric convection response to changing interplanetary magnetic field conditions using the assimilative mapping of ionospheric electrodynamics technique, J. Geophys. Res., 103, 4023, 1998.

Ridley, A. J., G. Lu, C. R. Clauer, and V. O. Papitashvili, Reply, J. Geophys. Res., 104, 4393, 1999.

Roelof, E. C., and D. G. Sibeck, Magnetopause shape as a bivariate function of interplanetary magnetic field $B_{Z}$ and solar wind dynamic pressure, J. Geophys. Res., 98, 21421, 1993.

Ruohoniemi, J. M., and R. A. Greenwald, The response of highlatitude convection to a sudden southward IMF turning, Geophys. Res. Lett., 25, 2913, 1998.

Ruohoniemi, J. M., R. A. Greenwald, O. de la Beaujardière, and M. Lester, The response of the high-latitude dayside ionosphere to an abrupt northward transition in the IMF, Ann. Geophysicae, 11, 544, 1993.

Saunders, M., M. P. Freeman, D. J. Southwood, S. W. H. Cowley, M. Lockwood, J. C. Samson, C. J. Farrugia, and T. J. Hughes, 
Dayside ionospheric convection changes in response to longperiod interplanetary magnetic field oscillations: determination of the ionospheric phase velocity, J. Geophys. Res., 97, 19373, 1992.

Taylor, J. R., T. K. Yeoman, M. Lester, M. J. Buonsanto, J. L. Scali, J. M. Ruohoniemi, and J. D. Kelly, Ionospheric convection during the magnetic storm of 20-21 March 1990, Ann. Geophysicae, 12, 1174, 1994.
Todd, H., S. W. H. Cowley, M. Lockwood, D. M. Willis, and H. Lühr, Response time of the high-latitude dayside ionosphere to sudden changes in the north-south component of the IMF, Planet. Space Sci., 36, 1415, 1988.

Weimer, D. R., Models of the high-latitude electric potentials derived with a least error fit of spherical harmonic coefficients, J. Geophys. Res., 100, 19595, 1995. 